\title{
SUMOylation targets shugoshin to stabilize sister kinetochore biorientation
}

Authors: Xue Bessie $\mathrm{Su}^{1}$, Menglu Wang ${ }^{1}$, Claudia Schaffner ${ }^{1}$, Dean Clift ${ }^{1,2}$, Olga O. Nerusheva $^{1}$, Triin Tammasalu ${ }^{3}$, Andreas Wallek ${ }^{4}$, Yehui $\mathrm{Wu}^{4}$, David A. Kelly ${ }^{1}$, A. Arockia Jeyaprakash ${ }^{1}$, Zuzana Storchova ${ }^{4,5}$, Ronald Hay ${ }^{3}$, Adèle L. Marston ${ }^{1 *} \dagger$

\section{Affiliations:}

${ }^{1}$ Wellcome Centre for Cell Biology, Institute of Cell Biology, University of Edinburgh, Edinburgh, UK. ${ }^{2}$ Laboratory of Molecular Biology, Medical Research Council, Cambridge, UK. ${ }^{3}$ Centre for Gene Regulation and Expression, University of Dundee, Dundee, UK. ${ }^{4}$ MPI Biochemistry, Am Klopferspitz 18, Martinsried, Germany. ${ }^{5}$ Paul Ehrlich Str 24, TU Kaiserslautern, Germany.

*Correspondence should be addressed to A.L.M. E-mail: adele.marston@ed.ac.uk $\dagger$ Lead contact 


\section{Abstract}

2 The accurate segregation of chromosomes during mitosis relies on the attachment of

3 sister chromatids to microtubules from opposite poles, called biorientation. Sister

4 chromatid cohesion resists microtubule forces, generating tension which provides the

5 signal that biorientation has occurred. How tension silences the surveillance pathways

6 that prevent cell cycle progression and correct erroneous kinetochore-microtubule

7 remains unclear. Here we identify SUMOylation as a mechanism that promotes

8 anaphase onset upon biorientation. SUMO ligases modify the tension-sensing

9 pericentromere-localized chromatin protein, shugoshin, to stabilize bioriented sister

10 kinetochore-microtubule attachments. In the absence of SUMOylation, Aurora B

11 kinase removal from kinetochores is delayed. Shugoshin SUMOylation prevents its

12 binding to protein phosphatase $2 \mathrm{~A}$ (PP2A) and release of this interaction is important

13 for stabilizing sister kinetochore biorientation. We propose that SUMOylation

14 modulates the kinase-phosphatase balance within pericentromeres to inactivate the

15 error correction machinery, thereby allowing anaphase entry in response to

16 biorientation. 


\section{Introduction}

Mitosis divides the nucleus to produce two genetically identical daughter cells. Prior to mitosis, DNA replication produces sister chromatids, linked together by the cohesin complex. Sister chromatids are aligned at metaphase, thus allowing microtubule spindles to be captured by kinetochores assembled on centromeres. The correct form of attachment is termed 'biorientation', meaning that the kinetochores on the two sister chromatids are attached to microtubules emanating from opposite spindle poles.

Biorientation creates tension, because cohesin holding sister chromatids together resists the pulling force of microtubules [1]. The fulfilment of biorientation allows securin degradation and, consequently, the activation of the protease separase, which cleaves cohesin, triggering sister chromatid separation (reviewed in [2]).

The conserved shugoshin protein plays key roles in promoting biorientation in mitosis and preventing cell cycle progression where biorientation fails $[3,4]$. Budding yeast possesses a single shugoshin gene, SGO1. Sgol localizes to both the core $\sim 125 \mathrm{bp}$ centromere, where the kinetochore resides, and the surrounding $\sim 20 \mathrm{~kb}$ cohesin-rich chromosomal region called the pericentromere [5]. The kinetochorelocalized Bub1 kinase promotes Sgol enrichment at the pericentromere through phosphorylation of S121 on histone H2A [6-8]. Sgo1, in turn, recruits condensin and protein phosphatase $2 \mathrm{~A}, \mathrm{PP} 2 \mathrm{~A}-\mathrm{Rts} 1$, to the pericentromere and maintains the chromosome passenger complex (CPC) containing Aurora B kinase at centromeres during mitosis $[9,10]$. Condensin at pericentromeres is thought to bias the conformation of the sister chromatids to favour biorientation. The $\mathrm{CPC}$ recognizes erroneous microtubule-kinetochore attachments and destabilizes them, thereby maintaining the activity of the spindle assembly checkpoint (SAC) to prevent anaphase entry (reviewed in [11]). In vertebrate cells, PP2A-B56 protects cohesin in pericentromeres from removal by the so-called prophase pathway, which removes cohesin through a non-proteolytic mechanism that is independent of separase $[12,13]$. In budding yeast, PP2A-Rts1 is recruited by shugoshin despite the absence of the prophase pathway $[9,10,14]$. Instead, PP2A-Rts1 has been implicated in ensuring the equal segregation of sister chromatids during mitosis, since mutants failing to recruit PP2A-Rts1 to the centromere are unable to respond to a lack of inter-sister kinetochore tension and mis-segregate chromosomes upon recovery [9,14].

Sgo1 both directs and responds to cell cycle cues as chromosomes establish and achieve biorientation, upon which anaphase entry is triggered. A key signal that 
biorientation has occurred is the tension-dependent removal of Sgo1 from the pericentromere during metaphase, resulting in the delocalization of its effectors, PP2A-Rts1, condensin and the CPC [8,14]. Upon anaphase I onset, Sgol is ubiquitinated and degraded by APC/C-Cdc20 [14,15]. Similarly, human shugoshin is degraded in anaphase as a result of APC/C-Cdc20 activity [16]. Shugoshin can be stabilized by mutation of its APC-Cdc20-dependent destruction sequence in both yeast and human cells, however, this does not impair the metaphase-anaphase transition $[13,14,17]$. Nevertheless, there is evidence that Sgol inactivation is important, since $S G O 1$ overexpression results in a pronounced metaphase delay and a block to cohesin cleavage [18]. Since the Sgo1-induced metaphase delay is abrogated by deletion of $B U B 1$, it is likely that Sgo1 must be localized at the pericentromere to prevent anaphase onset [18]. Accordingly, we showed that Sgol and its associated proteins are released from centromeres upon sister chromatid biorientation and tension [8]. Potentially, the temporal separation between Sgol removal from each pericentromere and its later degradation could ensure the reversibility of the mechanism that monitors biorientation right up until the moment that the commitment to anaphase is made. However, this model poses a conundrum: with the initiation of cohesin cleavage at anaphase onset, tension between sister kinetochores is lost, which could lead to re-activation of the error correction and biorientation pathways. What is more, it was unclear whether Sgo1- associated PP2A-Rts1 and CPC depart from centromeres simply by interacting with Sgo1, or by a more sophisticated mechanism involving finely orchestrated interplay between kinase and phosphatase activities.

Here, we identify Sgo1 SUMOylation as a mechanism that inactivates the pericentromeric signalling hub and thereby ensures timely anaphase onset. Small ubiquitin-like modifier (SUMO) is a $12 \mathrm{kDa}$ protein that is covalently added to lysine residues of SUMO substrates. SUMOylation is carried out by the sequential activities of E1 activation enzyme, E2 conjugation enzyme and E3 ligase [19]. We isolated the E3 SUMO ligase, Siz2, as a negative regulator of Sgo1 through a genetic screen. We show that Sgo1 is SUMOylated in a manner dependent on Siz2 together with its paralog, Siz1, and that a failure to SUMOylate Sgol causes a delay to anaphase onset. We provide evidence that Sgo1 SUMOylation modulates its interaction with PP2ARts 1 and facilitates the removal of CPC to stabilize sister chromatid biorientation, thereby enabling efficient entry into anaphase. 


\section{Results}

\section{SUMO ligases reverse the effects of SGO1 overexpression}

To identify negative regulators of Sgo1, we screened for high copy suppressors of the poor growth caused by $S G O 1$ overexpression [18]. We recovered a number of plasmids that improved the growth of cells carrying multiple copies of $S G O 1$ under galactose-inducible control ( $p G A L-S G O 1$ ) (Figure S1A; Table S1), including one carrying a $\sim 5 \mathrm{~kb}$ fragment containing truncated SLP1 and PUP1 together with full length ISN1 and SIZ2 (Figure 1A). SIZ2, encoding one of three budding yeast SUMO E3 ligases and sharing functional redundancy with its paralog, Siz1, is an attractive candidate for an Sgo1 antagonist, since Siz1/Siz2 have functions in chromosome segregation and cell division (Johnson and Gupta, 2001; Makhnevych et al., 2009; Montpetit et al., 2006; Takahashi et al., 2006). We used live cell imaging to confirm that overexpression of SIZ2 (by placement under control of the copper-inducible promoter, $p C U P 1-S I Z 2)$ counteracts the delay in metaphase caused by SGO1 overexpression (Figure 1B). Metaphase duration, estimated as the time between spindle pole body separation (emergence of two Spc42-tdTomato foci) and anaphase onset (dispersal of Cdc14-GFP from the nucleolus), was markedly reduced in $p G A L$ SGO1 $p C U P 1-S I Z 2$ cells compared to $p G A L-S G O 1$ cells (Figure 1C). These findings suggest that Siz1/Siz2 promote anaphase onset by counteracting Sgo1. Indeed, siz1 $\Delta$ siz $2 \Delta$ cells show a prolonged metaphase, measured as the time between formation of a short metaphase spindle (Tub1-YFP) and Cdc14-GFP dispersal (Figure 1D and E;

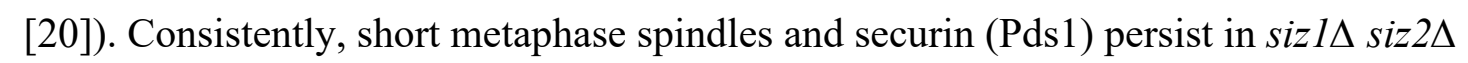
cells after synchronous release from a G1 arrest (Figure 1F and $1 \mathrm{G}$ ). Deletion of SGO1 in siz1 $\operatorname{siz} 2 \Delta$ cells partially rescued the metaphase delay, as judged by both decreased accumulation of short spindles and reduced Pds1 stabilization (Figure 1F and $1 \mathrm{G}$ ), as did deletion of CDC55 (Figure S1B), previously shown to rescue the metaphase delay of $p G A L-S G O 1$ [18], further indicating that ectopic Sgo1 activity is at least partially responsible for the metaphase delay of cells lacking SIZ1 and SIZ2.

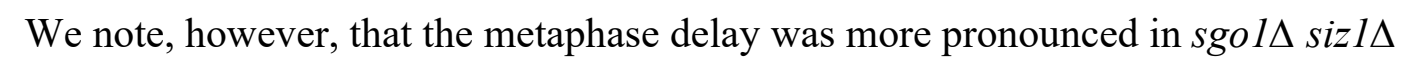
siz $2 \Delta$ cells than in $s g o 1 \Delta$ cells, indicating that additional factors prevent timely anaphase onset in the absence of Siz1-Siz2. We conclude that Siz1/Siz2 promote anaphase onset, in part by antagonising Sgo1. 
119 To determine whether Siz1/Siz2 might counteract Sgo1 by direct SUMOylation, we

120 assayed Sgo1-SUMO conjugates in vivo. Cells carrying SGO1-6HA and a plasmid

121 producing His-tagged yeast SUMO (7His-Smt3) were lysed under denaturing

122 conditions and SUMOylated proteins were isolated using nickel affinity

123 chromatography (Figure 2A). Anti-HA western blotting identified two slow-migrating

124 bands corresponding to SUMOylated Sgo1 in wild type but not siz1 $\triangle$ siz2 $\triangle$ cells

125 (Figure 2B). We further demonstrated direct SUMOylation of purified Sgol (Figure

126 S2) in vitro by either Siz1 or Siz2 (Figure 2C).

127 Sgol is recruited to the pericentromeric chromatin during S phase, released

128 into the nucleoplasm upon sister kinetochore biorientation at metaphase and degraded

129 in anaphase [8]. Sgo1 SUMOylation was maximal prior to anaphase onset, reflecting

130 Sgo1 abundance (Figure 2D). We assessed whether Sgo1 recruitment to

131 pericentromeric chromatin is important for its SUMOylation. Loss of Bub1 or

132 inactivation of its kinase activity which is required for phosphorylation of histone

133 H2A-S121 and Sgol recruitment to pericentromeres [6,21] greatly diminished Sgo1

134 SUMOylation (Figure 2E). Similarly, the sgo1-100 and sgo1-700 alleles, which carry

135 point mutations that delocalize Sgo1 from pericentromeres [10], also markedly

136 reduced Sgo1 SUMOylation (Figure 2E). To test whether Sgo1 removal from

137 pericentromeres in response to tension coincides with loss of SUMOylation, we

138 analysed cells arrested in metaphase (by depletion of CDC20) either in the presence (-

139 tension) or absence (+tension) of microtubule depolymerising drugs. Indeed, spindle

140 tension largely abrogated Sgo1 SUMOylation, though total Sgo1 levels were

141 comparable to the no tension condition (Figure 2F). We conclude that Sgo1

142 SUMOylation is promoted by its association with pericentromeres.

144 Sgo1 SUMOylation requires its coiled-coil

145 If Sgo1 SUMOylation mediates the effect of Siz1/Siz2 in the metaphase-anaphase

146 transition, a mutant that specifically abrogates Sgo1 SUMOylation, either by losing

147 the SUMOylation sites, or by reducing Sgo1's interaction with the E3 ligases, is

148 expected to recapitulate siz1 $\Delta$ siz2 $\Delta$ 's metaphase delay. With the aim to map the

149 SUMOylation sites, we performed mass spectrometry analysis of elutes from nickel

150 affinity chromatography identified Lys124 as an in vivo SUMOylation site (Table

151 S2). However, the sgol-K124R mutant retained high levels of SUMOylation,

152 suggesting the presence of additional SUMOylation sites (Figure 3A and B). As an 
153 alternative approach to mass spectrometry, we analysed a series of Sgo1 truncations

154 (Figure S3A). SUMOylation was abolished in Sgo1- $\Delta 2-208$ and reduced in Sgo1- $\Delta 2-$

155108 , suggesting that the N-terminal 108 amino acids are important for SUMOylation

156 together with K124R (Figure S3B). Further analysis revealed robust SUMOylation of

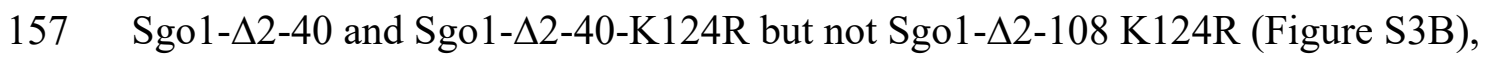

158 suggesting that SUMOylation requires amino acids 40-108. Interestingly, this region

159 encompasses the coiled-coil domain of budding yeast Sgo1, which is known to

160 directly bind PP2A-Rts 1 and is also important for maintaining CPC at the

161 centromeres (Verzijlbergen et al., 2014; Xu et al., 2009). We mutated the Lys residues

162 in this region in several combinations (Figure 3A). SUMOylation was progressively

163 reduced in the Sgo1-K56R K85R (Sgo1-2R), Sgo1-K56R K64R K70R K85R (Sgo1-

164 4R) and Sgo1-K56R K64R K70R K85R K124R (Sgo1-5R) mutants (Figure 3B). The

165 reduced SUMOylation of Sgo1-4R was recapitulated in the in vitro SUMOylation

166 assay (Figure 3C). Therefore, lysines in Sgol coiled-coil enable its SUMOylation and

167 may be direct targets of Siz1/Siz2. Moreover, mutation of these lysines specifically

168 reduced SUMOylation in Sgol both in vivo and in vitro, providing a tool to study the

169 effects of reduced Sgo1 SUMOylation in vivo.

170 Time course analysis and live cell imaging revealed a negligible or mild

171 metaphase delay in the sgo1-K124R and sgo1-2R mutants, respectively (Figure S3C

172 and D) and the sgol-2R mutation did not exacerbate the metaphase delay of sizl $\Delta$

173 siz2 $\Delta$ cells (Figure S3E). The sgol-4R mutant showed a more pronounced metaphase

174 delay (Figure 3D), but additional mutation of K124R (sgol-5R) largely abrogated this

175 delay (Figure S3F), suggesting adverse effects on Sgol protein function (see also

176 Figure 5B below). Therefore, we focused on sgol-4R for further analysis of the

177 effects of reduced Sgo1 SUMOylation.

Sgo1 is not a direct target of Slx5-Slx8-mediated ubiquitination

180 How might SUMOylation of Sgo1 reverse its inhibitory effects on anaphase onset?

181 Sgo1 was stabilized in cells lacking E3 ligases, Siz1 and Siz2 (Figure S1B), or where

182 E2 Ubc9 function was impaired (Figure S4A). Cells lacking the kinetochore-

183 associated Slx5/Slx8 SUMO-targeted ubiquitin ligase (STUbL) complex show

184 increased centromeric PP2A-Rts1 [22] and slx54 cells exhibit a metaphase delay in

185 which Sgo1 is stabilized (Figure 4A). This led us to test whether Sgo1-SUMO

186 conjugates are targeted for proteosomal degradation by Slx5/8 [23]. We measured the 
half-life of Sgo1 protein levels after transient expression of ectopic $p G A L-S G O 1$ in to wild type cells (Figure S4C), indicating that Siz1/Siz2 and Slx5/S1x8 promote Sgo1 degradation.

To examine whether Sgo1 could be a direct target of S1x5/Slx 8 we analysed ubiquitin conjugates in siz1 $\operatorname{siz} 2 \Delta$, slx $5 \Delta$ and sgol-4R cells. Since Sgo1 degradation also requires $\mathrm{APC} / \mathrm{C}$-dependent ubiquitination within a $\mathrm{C}$-terminal degradation box on Sgo1 [14], we also analyzed mutants lacking this region $(\Delta d b)$. Strikingly, Sgo1 ubiquitination was abolished in cells lacking the APC/C-dependent degradation box, but not detectably reduced in siz1 $\operatorname{siz} 2 \Delta$, slx $5 \Delta$ or sgol-4R cells, indicating that Sgo1 is unlikely to be a direct target of STUbL enzymes (Figure 4B). Consistently, Sgo1SUMO conjugates do not accumulate in slx5 cells (Figure 4C) and the half-life of Sgo1-2R was not increased over wild type Sgol (Figure S4D). Therefore, Slx5/S1x 8 regulates anaphase onset, but not by directly ubiquitinating Sgo1, and although Siz1/Siz2 and Slx5 affect Sgo1 stability, they do so indirectly, for example, by activating proteosome function [24].

Moreover, Sgol degradation appears dispensable for anaphase onset since sgol- $\Delta d b$ cells show no metaphase delay [14] and abolishing the APC/C recognition sites in Sgol did not exacerbate the metaphase delay of the sgol-2R mutant (sgol-2R $\Delta d b$, Figure S3D and S4E). Importantly, Sgol- $\Delta \mathrm{db}$ was SUMOylated to a similar extent to wild type Sgo1 (Figure 4C). Therefore, Sgo1 SUMOylation is able to

\section{Sumoylation does not promote Sgo1 removal from chromatin under tension}

212 Sgo1 is released from pericentromeres under tension [8] but whether this is critical for 213 the metaphase-anaphase transition remained unclear. To address this, we asked if

214 artificial tethering of Sgol to the kinetochore can prevent tension-dependent removal:

215 GFP-binding protein (GBP) -tagged Sgo1 was produced from a galactose-inducible

216 promoter (replacing endogenous Sgo1) in cells where the kinetochore protein Mtw1

217 was tagged with GFP (Mtw1-GFP) (Figure 4D). On its own, $p G A L-S G O 1-G B P$

218 expression caused a modest metaphase delay compared to the Mtw1-GFP control, 219 presumably due to mild overexpression (Figure 4D). However, Sgo1-GBP expression 220 in a strain producing Mtw1-GFP resulted in a severe delay in metaphase (Figure 4D). 
221 This delay required Sgo1 association with PP2A-Rts1 and/or CPC, because

222 kinetochore tethering of the Sgo1-3A mutant protein, which has lost these

223 interactions, resulted in a more modest metaphase delay (Figure 4D). Therefore,

224 kinetochore-associated Sgo1 prevents anaphase onset in a manner depending on its

225 ability to bind PP2A-Rts1 and/or CPC, showing that tension-dependent removal of

226 Sgol is critical for anaphase entry.

227 Based on these findings, we considered that Siz1/Siz2 may promote anaphase

228 entry by triggering the release of Sgo1 from chromosomes upon sister kinetochore

229 biorientation. In wild type metaphase-arrested cells, chromatin immunoprecipitation

230 followed by qPCR (ChIP-qPCR) showed that Sgol associates with a centromeric site

231 in the absence, but not presence, of spindle tension and this pattern was largely

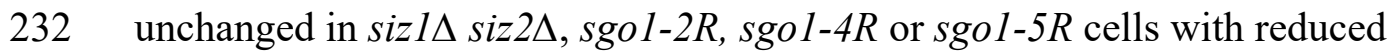

233 SUMOylation (Figure 4E and F; Figure S4F and G). We confirmed that

234 SUMOylation is dispensable for the tension-dependent release of Sgo1 during

235 anaphase by live cell imaging. In wild type cells, Sgo1-GFP first appeared as a bright

236 focus which dissociated upon splitting of Mtw1-tdTomato foci at metaphase and this

237 occurred with similar timing in siz1 $\Delta$ siz2 $\Delta$ cells (Figure 4G, Figure S4H). Instead,

238 the observed delay in anaphase entry occurred after bulk Sgol removal (Figure 4G).

239 Hence, although tension-dependent release of Sgol is critical for anaphase entry, this

240 occurs independently of Sgo1 SUMOylation.

Sgo1 SUMOylation is required for stabilizing biorientation

243 Our findings indicate that SUMOylation neither targets Sgo1 for STUbL-mediated

244 destruction nor does it facilitate Sgol removal under tension. To probe the mechanism

245 connecting Sgo1 SUMOylation to timely anaphase onset, we visualized the efficiency

246 of sister kinetochore biorientation in the Sgo1 SUMO mutants. We analysed the initial

247 establishment of sister kinetochore biorientation by monitoring the separation of sister

248 CEN4-GFP foci as spindles reformed after nocodazole wash-out, while maintaining a

249 metaphase arrest (by depletion of Cdc20, Figure 5A). In contrast to sgol-3A cells

250 which have impaired biorientation, likely due to impaired CPC binding[10], all

251 mutants with reduced Sgo1 SUMOylation except sgol-5R (siz1 $\operatorname{siz} 2 \Delta, \operatorname{sgol} 1-2 R$ and

252 sgol-4R) showed proficient sister kinetochore biorientation (Figure 5B). Therefore,

253 error correction and sister kinetochore biorientation pathways are functional in the

254 absence of Sgo1 SUMOylation. 
Next, we assessed the stability of biorientation in the SUMO mutants. Cells were released from nocodazole washout, and the separation of CEN4-GFP was monitored as cells progressed into anaphase. A single CEN4-GFP focus was observed initially, and two CEN4-GFP foci appeared upon attachment of sister kinetochores to microtubules from opposite poles. Stable attachment led to further separation of the two CEN4-GFP foci, which eventually segregated to opposite poles in anaphase (Figure 5C). In contrast, if attachments are unstable, the two CEN4-GFP foci reassociate prior to their splitting and segregation. In sgol $\Delta$ and sgol-3A mutants, which are defective in sensing and correcting attachment errors, the visualisation of two CEN4-GFP foci was delayed and the number of missegregation events was increased, but the levels of reassociation of two CEN4-GFP foci was similar to wild type (Figure 5D and E, Figure S5A and B). siz1 $\Delta$ siz2 $\Delta$ and sgo 1-4R mutants, in stark contrast, were proficient in the initial establishment of biorientation and did not show increased missegregation (Figure 5D and E, Figure S5A and B). Instead, both mutants showed $\sim 15 \%$ increase in the number of cells in which the two CEN4-GFP foci reassociated, indicative of unstable biorientation. We conclude that Sgo1 SUMOylation is important to maintain the bioriented state.

The metaphase delay in sizl $1 \Delta$ siz2 $\Delta$ is rescued by inactivating mutations in CPC/SAC Unstable biorientation in SUMO-deficient mutants is expected to generate unattached kinetochores and engage the SAC, potentially explaining the metaphase delay of these cells. Consistent with this idea, deletion of $M A D 2$ partially rescued the metaphase delay of siz1 1 siz2 $\Delta$ cells (Figure S6A). The CPC-dependent error correction pathway is likely responsible for the instability of kinetochore-microtubule interactons in the SUMO mutants because inhibition of the CPC component, Ipl1 (ipl1-as 1) also reduced the metaphase delay of siz $1 \Delta$ siz2 $\Delta$ cells (Figure 6A). Consistently, Sgo1 interaction with PP2A-Rts1 and/or CPC, is important for the metaphase delay in the absence of SUMOylation because both time course analysis (Figure S6B) and live cell imaging (Figure 6B) revealed that sgol-3A siz1 $\Delta$ siz2 $\Delta$ cells spent less time in

284 metaphase than siz1 $\Delta$ siz2 $\Delta$ cells. Interestingly, however, unlike Ipl1 (Figure 6A), the 285 PP2A regulatory subunit, Rts1, was largely dispensible for the metaphase delay of 286 siz1 $\Delta$ siz2 $\Delta$ cells (Figure S6C). Therefore, the CPC-dependent error correction 
287 pathway is responsible for the metaphase delay observed in the absence of Sgo1

288 SUMOylation.

Sgo1 SUMOylation promotes Ipl1 relocalization

291 The kinase activity of Ipl1 (Aurora B kinase) is required for error correction and Ipl1

292 is known to re-localize from centromeres to the spindle mid-zone upon the

293 establishment of biorientation [25,26]. Unstable biorientation in Sgo1 SUMOylation

294 mutants suggested that this removal may be incomplete. We monitored Ipl1-GFP and

295 its co-localization with Mtw1-tdTomato by imaging cells released from G1. In both

296 siz1s siz2s and sgol-4R, Ipl1 recruitment to the kinetochore-proximal regions

297 occurred normally (Figure 6C). However, as kinetochores separated, Ipl1-GFP

298 persisted close to kinetochores in the Sgo1 SUMO mutants (Figure 6C). Increased

299 centromeric Ipl1 was also measured by ChIP in SUMO-deficient cells arrested in

300 metaphase with kinetochores under tension (Figure S7A and B). Hence, Sgo1

301 SUMOylation facilitates the re-localization of Ipl1 from centromeres to prevent

302 persistent error correction and SAC activation.

Sgo1 SUMOylation is incompatible with PP2A-Rts1 binding

305 Interestingly, the coiled-coil domain of Sgo1 is both required for its SUMOylation

306 (Figure 3) and for PP2A-Rts1 binding [10,27]. This raised the question of whether

307 Sgo1 SUMOylation also impacts PP2A-Rts1 binding. Sgo1 SUMOylation was

308 increased in the Sgo1-3A mutant (Figure 7A), suggesting that PP2A-Rts1 binding

309 normally dampens Sgo1 SUMOylation. Structural modelling of budding yeast Sgo1-

310 PP2A-Rts1 interaction revealed that PP2A-Rts1 binding to Sgo1 would be

311 incompatible with SUMOylation on these residues (Figure S7C). We used an in vitro

312 binding assay to test the effects of Sgo1 SUMOylation on Rts1 binding. Purified Sgo1

313 was SUMOylated on beads in vitro, beads were stringently washed to remove

314 components of the SUMO reaction and subsequently incubated with cell-free extract

315 from sgold Rts1-9Myc cells. This revealed that, as expected, Rts1-9Myc bound

316 robustly to unSUMOylated Sgo1, however Rts1 binding was greatly reduced by Sgo1

317 SUMOylation, consistent with the prediction that SUMOylation and PP2A-Rts1

318 binding are mutually exclusive (Figure 7B). Similarly, immunoprecipitated Rts1-

319 9Myc bound in vitro SUMOylated Sgol less well than unSUMOylated Sgo1 (Figure

320 7C). Analysis of the SUMO-deficient Sgo1-4R protein showed that, unexpectedly, 
321 binding of Rts1 was reduced to a similar extent to Sgo1-3A, even in the absence of 322 SUMOylation (Figure 7B). Despite the fact that both mutants fail to bind PP2A-Rts1, 323 they result in very different outcomes in vivo: while sgol-3A shows defective initial 324 biorientation of sister kinetochores and chromosome mis-segregation after nocodazole 325 washout, sgo1-4R does not (Figure 5B and E). Conversely, unstable sister kinetochore 326 biorientation and a metaphase delay is observed in sgol-4R but not sgol-3A cells 327 (Figure 3D, Figure 5D, Figure 6B, Figure S6B). This indicates that a failure to bind 328 PP2A-Rts1 cannot be the primary cause of these defects. Instead, sister kinetochore 329 biorientation and chromosome segregation defects in sgol-3A cells are attributed to 330 defective CPC maintenance at kinetochores [10] while, conversely, our data indicate 331 that CPC persists at kinetochores in $s g o 1-4 R$ cells causing unstable biorientation and a 332 metaphase delay (Figure 6C). Collectively, these data indicate that the ability of Sgo1 333 to bind and release CPC underlies the establishment and stabilization of biorientation, 334 respectively (Figure S7D).

Dissociation of shugoshin and PP2A-Rts 1 stabilizes sister kinetochore biorientation

337 To understand the importance of the Sgo1-PP2A-Rts1 interaction in stabilizing sister

338 kinetochore biorientation, we asked whether tethering of Rts1 to wild type Sgo1 or 339 Sgo1-4R could rescue the instability of attachments. Although forcing Rts1 340 interaction with Sgo1-4R throughout the cell cycle did not affect initial biorientation 341 (Figure 7D), this state was unstable, as judged by the increased number of cells 342 switching between two and one CEN4-GFP dots (Figure 7E), though chromosome 343 segregation was ultimately successful in the majority of cells (Figure 7F). This further 344 confirms that loss of Rts1 binding is not the cause of unstable sister kinetochore 345 biorientation in sgol-4R cells. However, interestingly, forced interaction between 346 Rts 1 and wild type Sgo1 also resulted in frequent switches (Figure 7E). Therefore, 347 release of the Sgo1-Rts 1 interaction is important to stabilize bioriented sister 348 kinetochore-microtubule attachments. Together with our finding that PP2A-Rts1

349 binding is incompatible with Sgo1 SUMOylation, this suggests that PP2A-Rts1 350 dissociation as a result of Sgol SUMOylation, is also important to stabilize 351 biorientation. 
355 Starting with an unbiased genetic screen we have identified SUMO ligases as

356 negative regulators of the pericentromeric hub that responds to a lack of tension

357 between kinetochores. Sgo1, the central pericentromeric adaptor protein is one key

358 target of the Siz1/Siz2 SUMO ligases. Kinetochore-microtubule interactions are

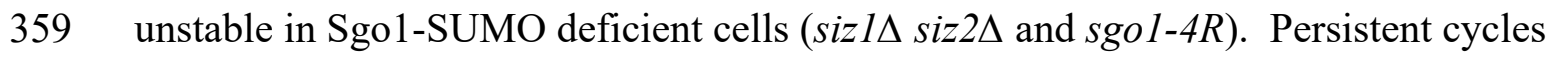

360 of kinetochore detachment and re-attachment engage the SAC, explaining why a

361 failure to SUMOylate Sgo1 results in a metaphase delay. Consistently, we find that

362 inactivation of components of the error correction pathway (Ip11, Mad2) advanced

363 anaphase timing in $\operatorname{siz} 1 \Delta$ siz2 $\Delta$ cells.

364 Sgol inactivation and removal from kinetochores is essential for timely

365 anaphase entry ([18], Figure 4D) and here we have identified one mechanism that

366 contributes to this inactivation. However, Sgol also prevents anaphase onset by

367 inhibiting separase independently of securin [18]. PP2A-Cdc55 dependent

368 dephosphorylation of separase and, potentially also cohesin itself is likely to underlie

369 this inhibition [28,29]. Notably, ZDS2, a negative regulator of PP2A-Cdc55 [30], was

370 also isolated in our screen along with HOS3, the cohesin deacetylase [31-33]

371 indicating that further mechanisms await discovery.

SUMOylation of Sgol ensures efficient entry into anaphase

374 How does Sgo1 SUMOylation regulate anaphase entry? We found that Sgo1

375 SUMOylation is dispensable for its tension-dependent release from pericentromeres

376 and that, although SUMOylation promotes Sgol degradation indirectly, this is not

377 required for efficient anaphase entry. Instead, our work suggested that Sgo1

378 SUMOylation likely promotes anaphase entry by silencing the error correction

379 process, as biorientation was highly unstable in Sgo1 SUMO-deficient mutants

380 (Figure 5D). Remarkably, we found that Ipl1 removal from kinetochores was

381 incomplete in the Sgo1 SUMO mutants (Figure 6C), suggesting a key role of this

382 modification in modulating the subcellular localization of Ipl1.

383 Meanwhile, we showed that Rts1 binds preferentially to unSUMOylated Sgo1,

384 and that tethering Sgo1 to Rts1 destabilized biorientation in a similar way as the Sgo1

385 SUMO mutants. These findings suggest that Sgo1 SUMOylation-mediated Rts 1

386 dissociation has an important role in stabilizing microtubule-kinetochore attachment.

387 Interestingly, PP2A-B56 dampens the effects of Aurora B to allow initial attachments

388 in human cells [34-36], suggesting a potential explanation for our observations. 
389 Overall, our findings indicate that SUMOylation modulates the kinase-phosphatase

390 balance at the kinetochore to dampen CPC activity and allow initial kinetochore-

391 microtubule attachments (Figure 7G). Different mutants affect this balance in distinct

392 ways, leading to the observed outcomes on the establishment and stabilization of

393 biorientation (Figure S7D).

395 SUMOylation - a generalized mechanism of centromere regulation with implications

396 for disease?

397 Accumulating evidence indicates that SUMOylation might play a specific role at

398 centromeres to fine-tune chromosome segregation. The SUMO isopeptidase

399 Ulp2/Smt4 is important for maintenance of cohesion specifically at centromeres, in

400 part through regulating Topoisomerase II [37]. The Pds5 subunit of cohesin is also

401 known to prevent polySUMOylation of cohesin [38] and centromeric cohesin may be

402 particularly susceptible since it lacks Pds5 [39]. PIAS SUMO ligases are known to be

403 localized at centromeres in vertebrate mitotic cells and oocytes [40-42]. Moreover, the

404 SUMO pathway is required to prevent cohesion loss during meiosis II, which

405 centrally requires Sgo2-PP2A, and it is conceivable that modulation of the PP2A-

406 Sgol interaction as we find in yeast underlies these observations in mouse oocytes

407 [43]. Indeed, global studies found that shugoshins are SUMOylated in fission yeast

408 and human cells, though the function has yet to be tested [44,45]. Furthermore, there

409 is ample evidence that CPC function may be subject to regulation by SUMOylation.

410 Aurora B SUMOylation in Xenopus and human cells was shown to promote its

411 enrichment at centromeres and it was proposed that this modification may serve as a

412 reversible mechanism to dampen Aurora B kinase activity [46], while in C. elegans

413 meiosis the localization of both Aurora B and Bub1 kinase are influenced by

414 SUMOylation [47-49]. This suggests that SUMO may have a general role in

$415 \mathrm{CPC} /$ error correction pathways and we speculate that multi-lateral SUMO-SIM

416 interactions [50] enable the coordinated re-localization of surveillance factors.

417 Notably, mis-regulation of the SUMO pathway is wide-spread in different cancers

$418[51,52]$. Potentially, reductions in chromosome segregation fidelity caused by SUMO

419 malfunction at centromeres, as we show here, could be a contributing factor in driving

420 these malignant states. 
423

424

425

426

427

428

429

430

431

432

433

434

435

436

437

438

439

440

441

442

443

444

445

446

447

448

449

450

451

452

453

454

455

456

457

458

459

460

461

462

463

We are grateful to Yoshiko Kikuchi and Helle Ulrich for yeast strains and plasmids, respectively, to Weronika Borek for help with R programming. We thank Lorraine Pillus, Emily Petty, Federico Pelisch and Andrew Goryachev for helpful discussions and comments on the manuscript. This work was funded by Wellcome through Senior Research Fellowships to AM [[107827]] and AAJ [202811], and core funding for the Wellcome Centre for Cell Biology [203149].

\section{Author contributions}

Conceptualization, A.L.M., X.B.S., C.S., L.W., O.N., D.C., A.W. and Z.S.;

Methodology, X.B.S, M.W., C.S., O.N., T.T. and R.H. Investigation: X.B.S., M.W., C.S., D.C., O.N., A.W., T.T. and Y.W. Software: D.A.K. Writing - original draft, X.B.S.. and A.L.M.; Writing - Review and Editing, all authors; Visualization, X.B.S., A.A.J. and A.L.M.; Supervision, A.L.M., R.H. and Z.S.; Funding acquisition, A.L.M.

\section{Declaration of interests}

The authors declare no competing interests.

\section{References}

[1] Tanaka TU. Kinetochore-microtubule interactions: steps towards bi-

orientation. Embo J 2010;29:4070-82. doi:10.1038/emboj.2010.294

[2] Marston AL. Chromosome segregation in budding yeast: sister chromatid cohesion and related mechanisms. Genetics 2014;196:31-63. doi:10.1534/genetics.112.145144.

[3] Indjeian VB, Stern BM, Murray AW. The centromeric protein Sgo1 is required to sense lack of tension on mitotic chromosomes. Science 2005;307:130-3.

[4] Indjeian VB, Murray AW. Budding yeast mitotic chromosomes have an intrinsic bias to biorient on the spindle. Curr Biol 2007;17:1837-46.

[5] Kiburz BM, Reynolds DB, Megee, P. C., Marston AL, Lee BH, Lee TI, et al. The core centromere and Sgol establish a 50-kb cohesin-protected domain around centromeres during meiosis I. Genes Dev 2005; 19:3017-30.

[6] Fernius J, Hardwick KG. Bub1 kinase targets Sgo1 to ensure efficient chromsoome biorientation in budding yeast mitosis. PLoS Genet 2007;3:e213.

[7] Yamagishi Y, Honda T, Tanno Y, Watanabe Y. Two histone marks establish the inner centromere and chromosome bi-orientation. Science 2010;330:23943. doi:10.1126/science.1194498.

[8] Nerusheva OO, Galander S, Fernius J, Kelly D, Marston AL. Tensiondependent removal of pericentromeric shugoshin is an indicator of sister chromosome biorientation. Genes Dev 2014;28:1291-309.

doi:10.1101/gad.240291.114. 


\begin{tabular}{|c|c|c|}
\hline $\begin{array}{l}464 \\
465\end{array}$ & [9] & $\begin{array}{l}\text { Peplowska K, Wallek AU, Storchová Z. Sgol regulates both condensin and } \\
\text { ipl1/aurora B to promote chromosome biorientation. PLoS Genet }\end{array}$ \\
\hline 466 & & 2014;10:e1004411. doi:10.1371/journal.pgen.1004411. \\
\hline 467 & \multirow[t]{3}{*}[10]{} & $\begin{array}{l}\text { Verzijlbergen KF, Nerusheva OO, Kelly D, Kerr A, Clift D, de Lima Alves F, } \\
\text { et al. Shugoshin biases chromosomes for biorientation through condensin }\end{array}$ \\
\hline 69 & & recruitment to the pericentromere. Elife $2014 ; 3: \mathrm{e} 01374$. \\
\hline 470 & & doi:10.7554/eLife. 01374 \\
\hline 471 & \multirow[t]{3}{*}{ [11] } & Foley EA, Kapoor TM. Microtubule attachment and spindle assembly \\
\hline 72 & & checkpoint signalling at the kinetochore. Nat Rev Mol Cell Biol 2013;14:25- \\
\hline 473 & & 37. doi:10.1038/nrm3494. \\
\hline 474 & \multirow[t]{3}{*}{ [12] } & McGuinness BE, Hirota T, Kudo NR, Peters JM, Nasmyth K. Shugoshin \\
\hline 75 & & prevents dissociation of cohesin from centromeres during mitosis in \\
\hline 476 & & vertebrate cells. PLoS Biology 2005;3:e86. \\
\hline 477 & \multirow[t]{3}{*}{ [13] } & Liu H, Rankin S, Yu H. Phosphorylation-enabled binding of SGO1-PP2A to \\
\hline 478 & & cohesin protects sororin and centromeric cohesion during mitosis. Nature Cell \\
\hline 479 & & Biology 2013;15:40-9. doi:10.1038/ncb2637. \\
\hline 480 & \multirow[t]{3}{*}{ [14] } & Eshleman HD, Morgan DO. Sgo1 recruits PP2A to chromosomes to ensure \\
\hline 81 & & sister chromatid bi-orientation during mitosis. Journal of Cell Science \\
\hline 482 & & 2014;127:4974-83. doi:10.1242/jcs.161273. \\
\hline 483 & \multirow[t]{3}{*}[15]{} & Marston AL, Tham WH, Shah H, Amon A. A genome-wide screen identifies \\
\hline 84 & & genes required for centromeric cohesion. Science 2004;303:1367-70. \\
\hline 485 & & doi:10.1126/science. 1094220 \\
\hline 486 & {$[16]$} & Salic A, Waters JC, Mitchison TJ. Vertebrate shugoshin links sister \\
\hline 487 & & centromere cohesion and kinetochore microtubule stability in mitosis. Cell \\
\hline 488 & & $2004 ; 118: 567-78$ \\
\hline 489 & \multirow[t]{4}{*}{ [17] } & Karamysheva Z, Díaz-Martínez LA, Crow SE, Li B, Yu H. Multiple \\
\hline 490 & & anaphase-promoting complex/cyclosome degrons mediate the degradation of \\
\hline 491 & & human Sgo1. The Journal of Biological Chemistry 2009;284:1772-80. \\
\hline 492 & & doi:10.1074/jbc.M807083200. \\
\hline 493 & \multirow[t]{2}{*}{ [18] } & Clift D, Bizzari F, Marston AL. Shugoshin prevents cohesin cleavage by \\
\hline 494 & & PP2A(Cdc55)-dependent inhibition of separase. Genes Dev 2009;23:766-80. \\
\hline 495 & \multirow[t]{2}{*}{ [19] } & Hay RT. SUMO: a history of modification. Molecular Cell 2005;18:1-12. \\
\hline 496 & & doi:10.1016/j.molcel.2005.03.012. \\
\hline 497 & \multirow[t]{2}{*}[20]{} & Johnson ES, Gupta AA. An E3-like factor that promotes SUMO conjugation \\
\hline 498 & & to the yeast septins. Cell $2001 ; 106: 735-44$ \\
\hline 499 & \multirow[t]{3}{*}{ [21] } & Kawashima SA, Yamagishi Y, Honda T, Ishiguro K-I, Watanabe Y. \\
\hline 500 & & Phosphorylation of H2A by Bub1 prevents chromosomal instability through \\
\hline 501 & & localizing shugoshin. Science 2010;327:172-7. doi:10.1126/science.1180189. \\
\hline 502 & \multirow[t]{4}{*}[22]{} & van de Pasch LAL, Miles AJ, Nijenhuis W, Brabers NACH, van Leenen D, \\
\hline 503 & & Lijnzaad P, et al. Centromere binding and a conserved role in chromosome \\
\hline 504 & & stability for SUMO-dependent ubiquitin ligases. PLoS ONE 2013;8:e65628. \\
\hline 505 & & doi:10.1371/journal.pone. 0065628 . \\
\hline 506 & \multirow[t]{4}{*}{ [23] } & Schweiggert J, Stevermann L, Panigada D, Kammerer D, Liakopoulos D. \\
\hline 507 & & Regulation of a Spindle Positioning Factor at Kinetochores by SUMO- \\
\hline 508 & & Targeted Ubiquitin Ligases. Dev Cell 2016;36:415-27. \\
\hline 509 & & doi:10.1016/j.devcel.2016.01.011. \\
\hline 510 & \multirow[t]{4}{*}{ [24] } & Rao HBDP, Qiao H, Bhatt SK, Bailey LRJ, Tran HD, Bourne SL, et al. A \\
\hline  & & SUMO-ubiquitin relay recruits proteasomes to chromosome axes to regulate \\
\hline 1 & & meiotic recombination. Science $2017 ; 355: 403-7$. \\
\hline 813 & & ence.aaf640 \\
\hline
\end{tabular}


[25] Buvelot S, Tatsutani SY, Vermaak D, Biggins S. The budding yeast Ipl1/Aurora protein kinase regulates mitotic spindle disassembly. J Cell Biol 2003;160:329-39.

[26] Pereira G, Schiebel E. Separase regulates INCENP-Aurora B anaphase spindle function through Cdc14. Science 2003;302:2120-4. doi:10.1126/science.1091936.

[27] Xu Z, Cetin B, Anger M, Cho US, Helmhart W, Nasmyth K, et al. Structure and function of the PP2A-shugoshin interaction. Molecular Cell 2009;35:426-41. doi:10.1016/j.molcel.2009.06.031.

[28] Lianga N, Doré C, Kennedy EK, Yeh E, Williams EC, Fortinez CM, et al. Cdk1 phosphorylation of Esp1/Separase functions with PP2A and Slk19 to regulate pericentric Cohesin and anaphase onset. PLoS Genet 2018;14:e1007029. doi:10.1371/journal.pgen.1007029.

[29] Yaakov G, Thorn K, Morgan DO. Separase biosensor reveals that cohesin cleavage timing depends on phosphatase PP2A(Cdc55) regulation. Dev Cell 2012;23:124-36. doi:10.1016/j.devcel.2012.06.007.

[30] Queralt E, Uhlmann F. Separase cooperates with Zds1 and Zds2 to activate Cdc14 phosphatase in early anaphase. J Cell Biol 2008;182:873-83.

[31] Xiong B, Lu S, Gerton JL. Hos 1 is a lysine deacetylase for the Smc3 subunit of cohesin. Curr Biol 2010;20:1660-5. doi:10.1016/j.cub.2010.08.019.

[32] Beckouët F, Hu B, Roig MB, Sutani T, Komata M, Uluocak P, et al. An Smc3 acetylation cycle is essential for establishment of sister chromatid cohesion. Molecular Cell 2010;39:689-99. doi:10.1016/j.molcel.2010.08.008.

[33] Borges V, Lehane C, Lopez-Serra L, Flynn H, Skehel M, Rolef Ben-Shahar $\mathrm{T}$, et al. Hos 1 deacetylates Smc3 to close the cohesin acetylation cycle. Molecular Cell 2010;39:677-88. doi:10.1016/j.molcel.2010.08.009.

[34] Vallardi G, Allan LA, Crozier L, Saurin AT. Division of labour between PP2A-B56 isoforms at the centromere and kinetochore. Elife 2019;8:683. doi:10.7554/eLife.42619.

[35] Meppelink A, Kabeche L, Vromans MJM, Compton DA, Lens SMA. Shugoshin-1 Balances Aurora B Kinase Activity via PP2A to Promote Chromosome Bi-orientation. Cell Rep 2015;11:508-15. doi:10.1016/j.celrep.2015.03.052.

[36] Foley EA, Maldonado M, Kapoor TM. Formation of stable attachments between kinetochores and microtubules depends on the B56-PP2A phosphatase. Nature Cell Biology 2011;13:1265-71. doi:10.1038/ncb2327.

[37] Stephens AD, Snider CE, Bloom K. The SUMO deconjugating peptidase $\mathrm{Smt} 4$ contributes to the mechanism required for transition from sister chromatid arm cohesion to sister chromatid pericentromere separation. Cell Cycle (Georgetown, Tex 2015;14:2206-18. doi:10.1080/15384101.2015.1046656.

[38] DAmbrosio LM, Lavoie BD. Pds5 Prevents the PolySUMO-Dependent Separation of Sister Chromatids. Current Biology 2014;24:361-71. doi:10.1016/j.cub.2013.12.038.

[39] Petela N, Gligoris TG, Metson JS, Lee B-G, Voulgaris M, Hu B, et al. Multiple interactions between Scc1 and Scc2 activate cohesin's DNA dependent ATPase and replace Pds5 during loading 2017. doi:10.1101/205914. 
[40] Azuma Y, Arnaoutov A, Anan T, Dasso M. PIASy mediates SUMO-2 conjugation of Topoisomerase-II on mitotic chromosomes. Embo J 2005;24:2172-82. doi:10.1038/sj.emboj.7600700.

[41] Ban R, Nishida T, Urano T. Mitotic kinase Aurora-B is regulated by SUMO2/3 conjugation/deconjugation during mitosis. Genes Cells 2011;16:652-69. doi:10.1111/j.1365-2443.2011.01521.x.

[42] Ding Y, Kaido M, Llano E, Pendás AM, Kitajima TS. The Post-anaphase SUMO Pathway Ensures the Maintenance of Centromeric Cohesion through Meiosis I-II Transition in Mammalian Oocytes. Current Biology 2018;28:1661-4. doi:10.1016/j.cub.2018.04.019.

[43] Ding Y, Kaido M, Llano E, Pendás AM, Kitajima TS. The Post-anaphase SUMO Pathway Ensures the Maintenance of Centromeric Cohesion through Meiosis I-II Transition in Mammalian Oocytes. Curr Biol 2018;28:1661-4. doi:10.1016/j.cub.2018.04.019.

[44] Schimmel J, Eifler K, Sigurðsson JO, Cuijpers SAG, Hendriks IA, Verlaande Vries M, et al. Uncovering SUMOylation dynamics during cell-cycle progression reveals FoxM1 as a key mitotic SUMO target protein. Molecular Cell 2014;53:1053-66. doi:10.1016/j.molcel.2014.02.001.

[45] Nie M, Vashisht AA, Wohlschlegel JA, Boddy MN. High Confidence Fission Yeast SUMO Conjugates Identified by Tandem Denaturing Affinity Purification. Sci Rep 2015;5:14389. doi:10.1038/srep14389.

[46] Fernández-Miranda G, Pérez de Castro I, Carmena M, Aguirre-Portolés C, Ruchaud S, Fant X, et al. SUMOylation modulates the function of Aurora-B kinase. Journal of Cell Science 2010;123:2823-33. doi:10.1242/jcs.065565.

[47] Pelisch F, Bel Borja L, Jaffray EG, Hay RT. Sumoylation regulates protein dynamics during meiotic chromosome segregation in C. elegans oocytes. Journal of Cell Science 2019:jcs.232330. doi:10.1242/jcs.232330.

[48] Pelisch F, Tammsalu T, Wang B, Jaffray EG, Gartner A, Hay RT. A SUMODependent Protein Network Regulates Chromosome Congression during Oocyte Meiosis. Molecular Cell 2017;65:66-77.

doi:10.1016/j.molcel.2016.11.001.

[49] Davis-Roca AC, Divekar NS, Ng RK, Wignall SM. Dynamic SUMO remodeling drives a series of critical events during the meiotic divisions in Caenorhabditis elegans. PLoS Genet 2018;14:e1007626. doi:10.1371/journal.pgen.1007626.

[50] Jentsch S, Psakhye I. Control of nuclear activities by substrate-selective and protein-group SUMOylation. Annual Review of Genetics 2013;47:167-86. doi:10.1146/annurev-genet-111212-133453.

[51] Rabellino A, Andreani C, Scaglioni PP. The Role of PIAS SUMO E3-Ligases in Cancer. Cancer Res 2017;77:1542-7. doi:10.1158/0008-5472.CAN-162958.

[52] Seeler J-S, Dejean A. SUMO and the robustness of cancer. Nat Rev Cancer 2017;17:184-97. doi:10.1038/nrc.2016.143.

[53] Windecker H, Ulrich HD. Architecture and assembly of poly-SUMO chains on PCNA in Saccharomyces cerevisiae. Journal of Molecular Biology 2008;376:221-31. doi:10.1016/j.jmb.2007.12.008.

[54] Kelley LA, Mezulis S, Yates CM, Wass MN. The Phyre2 web portal for protein modeling, prediction and analysis. Nat. Protocols 10: 845-858. 2015. 



Figure 1. SUMO ligases promote Sgo1 inactivation to allow timely anaphase progression. (A) Overexpression of $S I Z 2$ rescues the slow growth phenotype of $S G O 1$ overexpression. A $p G A L-S G O 1$ (AMy870) strain carrying empty vector (AMp67), or with yEP13- (SLP1) ISN1 SIZ2 (PUP1) (AMp1435) was streaked onto medium containing galactose. (B and C) SIZ2 overexpression partially rescues the metaphase delay of SGO1-overexpressing cells. (B) Schematic of live cell imaging experiment. Cells carrying Spc42-tdTomato and Cdc14-GFP were synchronized in G1 in media containing $2 \%$ raffinose. $25 \mu \mathrm{M}$ copper sulfate was added to induce $p$ CUP1-SIZ2 expression. After releasing from $\mathrm{G} 1,0.2 \%$ galactose was added to induce $p G A L$ $S G O 1$ expression. The duration of metaphase was estimated by the time taken between the separation of the spindle pole bodies (two Spc42-tdTomato foci) and the dispersal of Cdc14-GFP from the nucleolus. (C) Metaphase duration is shown for wild type (AMy24115), $p$ GAL-SGO1 (AMy27596), $p$ GAL-SGO1 pCUP1-SIZ2 (AMy27738) and $p C U P 1-S I Z 2$ (AMy27952) strains. (D and E) Siz1 and Siz2 are required for timely anaphase onset. Metaphase duration was determined as the time between formation of a short bipolar spindle (YFP-Tub1) and release of Cdc14-GFP from the nucleolus from live cell imaging. (D) Schematics and representative images are shown. (E) Metaphase duration is shown for wild type (AMy24174) and siz1 $\Delta$ siz2 $\Delta$ (AMy24313) strains. (F) and (G) The metaphase delay of siz1 $\Delta$ siz2 $\Delta$ cells is

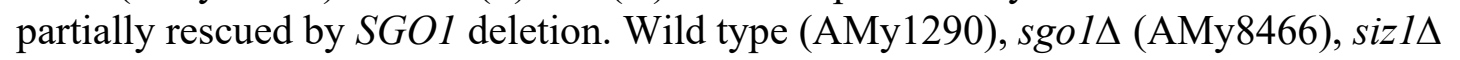

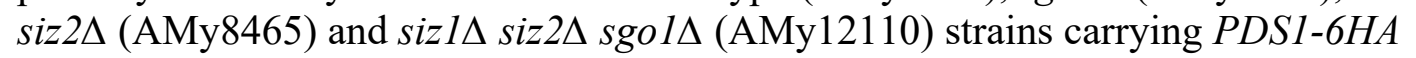
were released from a G1 arrest. Spindle morphology was scored after anti-tubulin immunofluorescence and the percentages of short (metaphase) spindles are shown (top) and Pds1 levels were analysed by anti-HA Western blot (bottom). Pgk1 is shown as a loading control. 


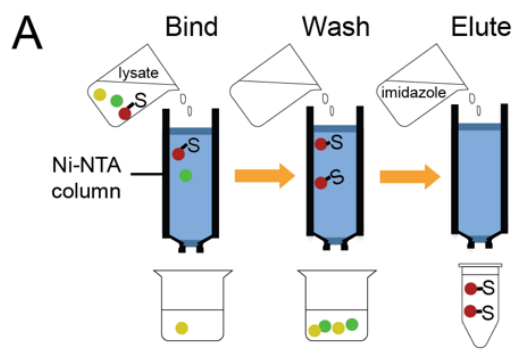

- $S=$ Substrates modified by 7 His-Smt3 (SUMO)

B

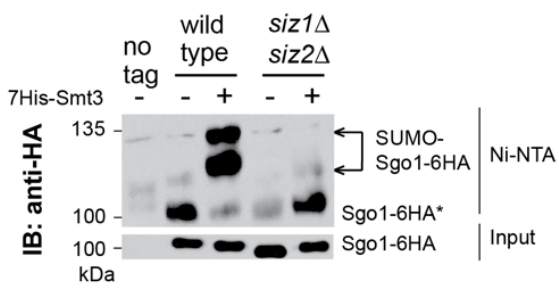

C

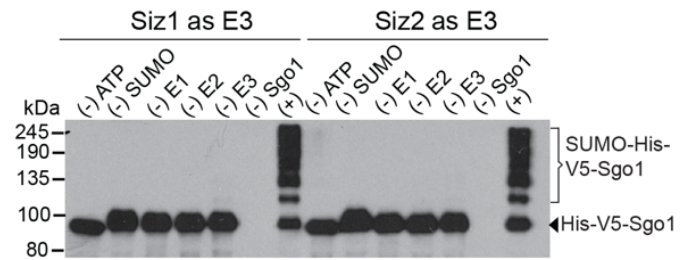

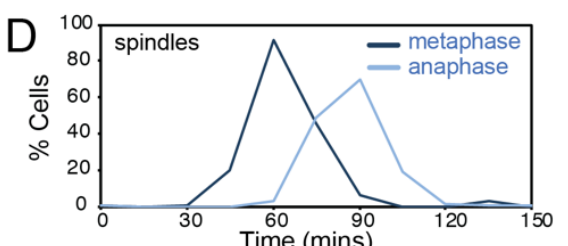


Figure 2. Sgo1 is SUMOylated, depending on its association with pericentromeres. (A and B) Sgo1 is SUMOylated in a Siz1/Siz2-dependent manner. (A) Scheme describing purification of SUMOylated proteins. (B) Extracts from untagged (AMy1176), SGO1-6HA (AMy906) and siz1 $\operatorname{siz} 2 \triangle$ SGO1-6HA (AMy7911) strains carrying empty vector (pRS426), or with $7 \times H I S-S M T 3$ (AMp773) were purified over Ni-NTA resin and anti-HA immunoblot was performed on both input and elute. Arrows and asterisks indicate SUMO-Sgo1-6HA and unmodified Sgo1-6HA, which binds non-specifically to the resin, respectively. (C) Sgol is SUMOylated by Siz1 and Siz2 in vitro. Purified Sgo1 was incubated with $1 \mu \mathrm{M}$ E1, E2, E3, SUMO and ATP or missing one component as indicated. Reaction was incubated at $30^{\circ} \mathrm{C}$ for $3 \mathrm{~h}$. (D) Sgo1 SUMOylation occurs in metaphase. Cells carrying SGO1-6HA and 7xHIS-SMT3 (AMy7655) were released from G1, harvested at the indicated intervals, and SUMOylation was analysed as described in (A). Cell cycle stage was monitored by scoring spindle morphology after anti-tubulin immunofluorescence. (E) Chromatin association promotes Sgo1 SUMOylation. Sgo1 SUMOylation was determined in wild type (AMy7654), bub1 1 (AMy10098), bub1-KD (catalytically inactive Bub1 kinase, AMy10102), sgo1-100 (AMy26334) and sgol-700 (AMy26336) strains. (F) Sgo1 SUMOylation is lost upon the establishment of tension between sister kinetochores. Cells carrying pMET-CDC20 and either 7xHIS-SMT3 (AM9641) or empty vector (AMy26342) were arrested in metaphase by depletion of Cdc20 either in the presence of benomyl and nocodazole (no tension) or DMSO (tension). 


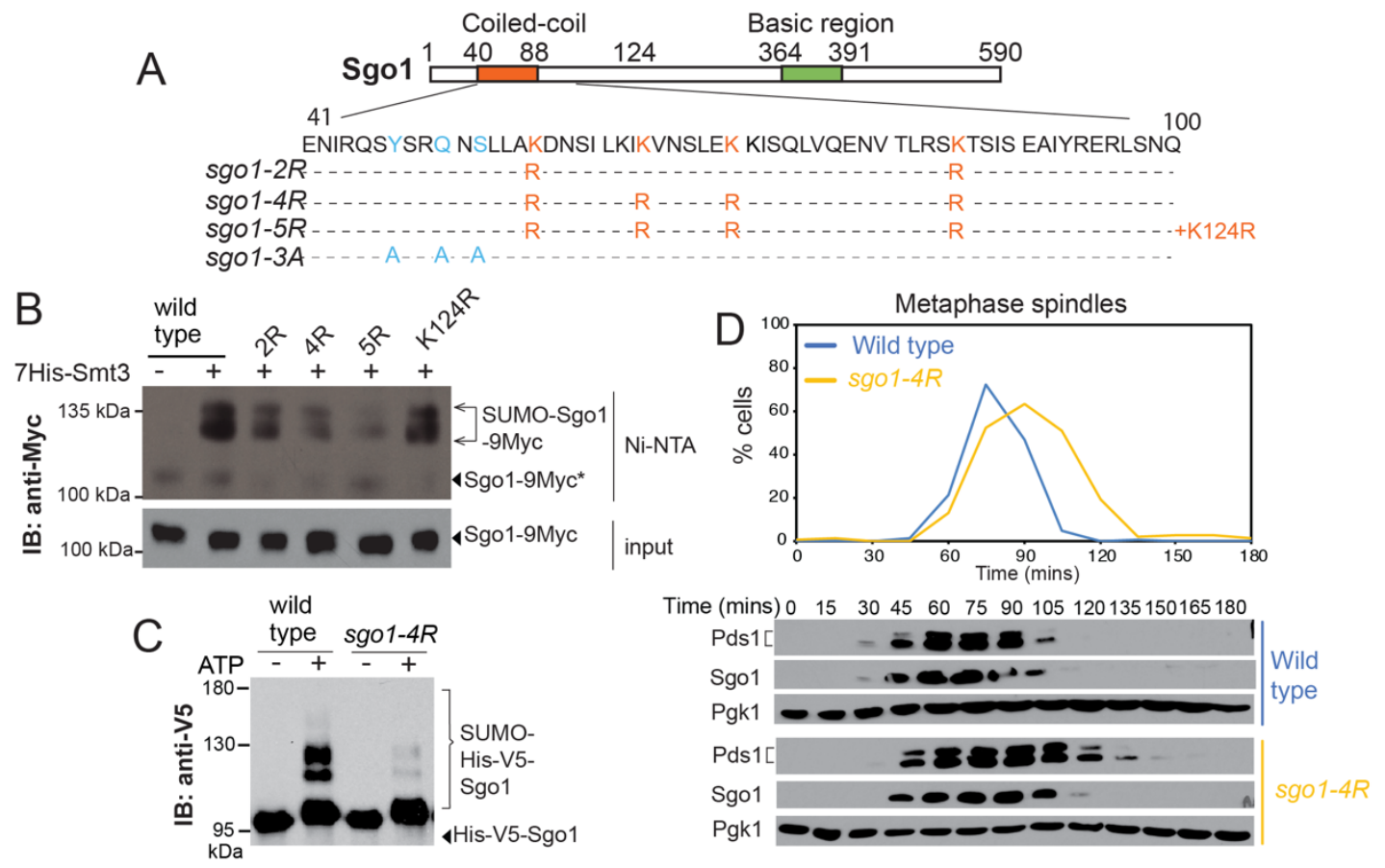

Figure 3 Sgo SUMOylation requires residues within its coiled-coil and is important for timely anaphase onset. (A) Schematic of Sgol showing the sequence of the coiledcoil domain (bottom) and residues mutated in the indicated mutants. (B) Sgol

SUMOylation requires residues K56, K64, K70, K85 and K124. Strains for in vivo SUMOylation analysis carried Sgo1-9Myc and were wild type (AMy24367), sgol$K 56 R K 85 R$ ('2R', AMy24299), sgol-K56R K64R K70R K85R ('4R', AMy23828), sgo1-K56R K64R K70R K85R K124R ('5R', AMy24371) and sgo1-K124R (AMy24369). (C) SUMOylation is reduced in vitro for the sgol-4R mutant. Purified Sgo1 and Sgo1-4R proteins were SUMOylated in vitro using $0.1 \mu \mathrm{M}$ E1-E3, in the presence or absence of ATP. (D) The sgo 1-4R mutant is delayed in metaphase. Cell cycle analysis of wild type (AMy8467) and sgol-K56R K64R K70R K85R-9Myc (AMy23934) strains carrying SGO1-9MYC and PDS1-3HA was performed as described in Figure 1F. 
A
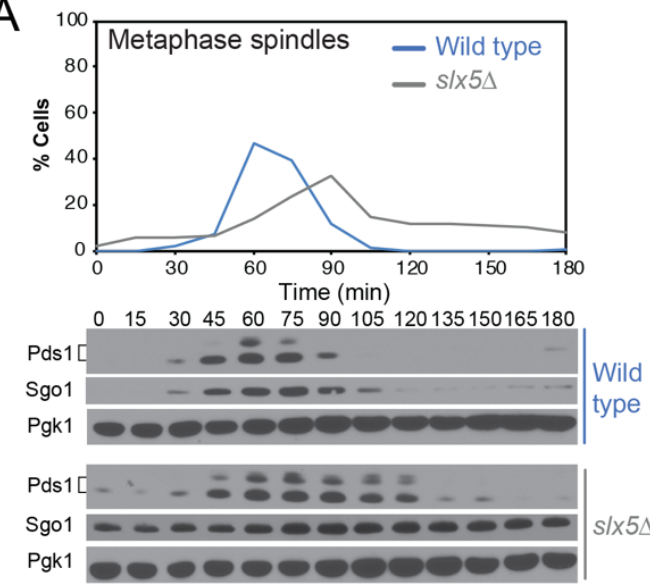

D
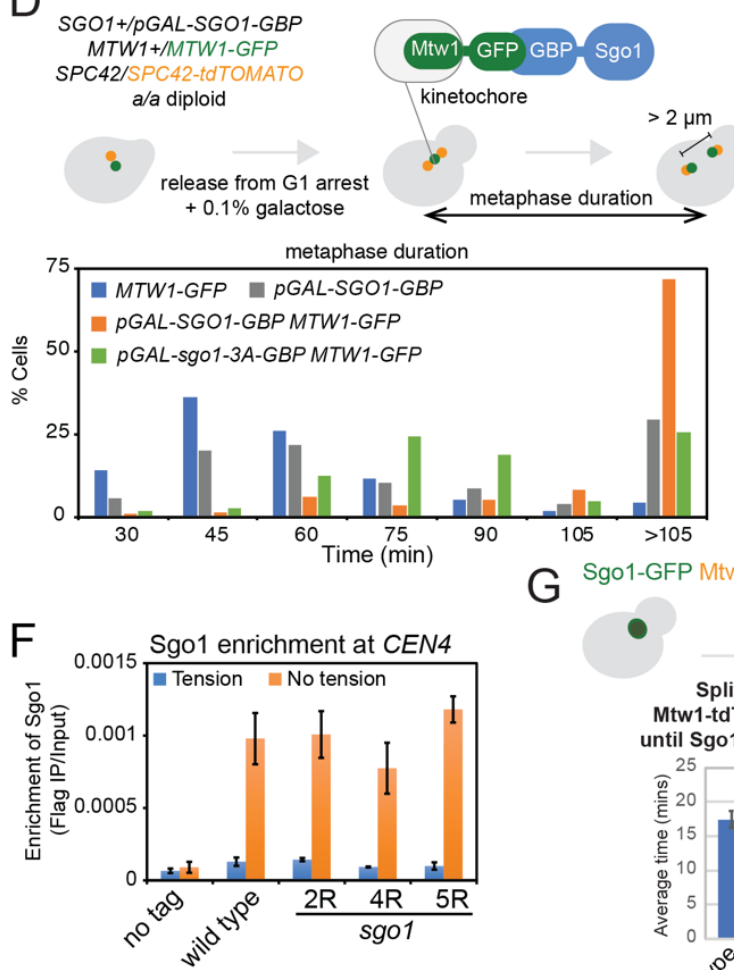

G

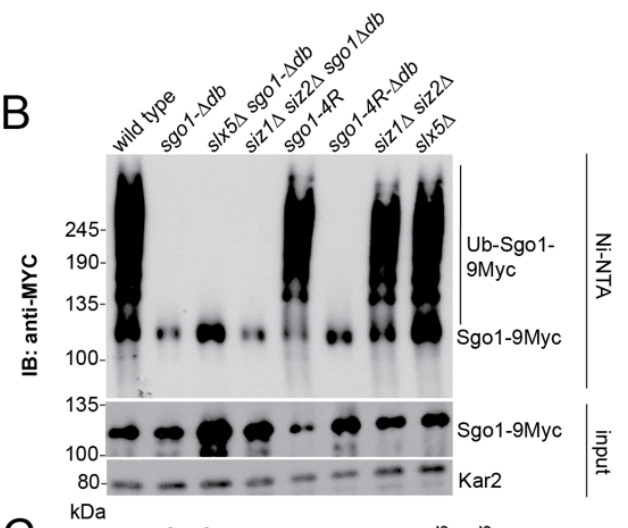

C

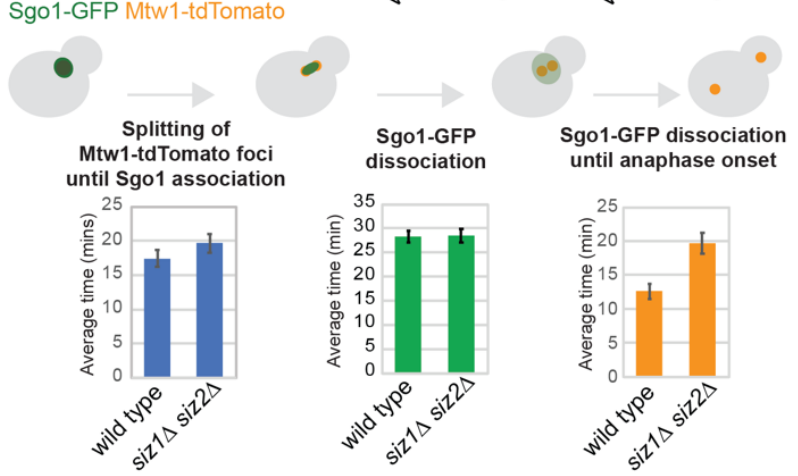

Figure 4. SUMOylation promotes anaphase onset independently of Sgo1 degradation or removal from pericentromeres. (A) slx $5 \Delta$ cells exhibit a metaphase delay and stabilize Sgo1. Wild type (AMy8467) and slx5 (AMy10981) strains carrying PDS1$3 H A$ and SGO1-9MYC were analysed as described in Figure 1F. (B) Sgol ubiquitination is dependent on its destruction box and independent of Sgo1 SUMOylation or Slx5/Slx8. Strains with $p G A L-S G O 1$ (AMy27029), $p G A L-s g o 1-\triangle d b$ (AMy27030), slx5 $p$ pAL-sgol- $\Delta d b$ (AMy27031), siz1 $\Delta$ siz2 $\Delta p G A L-s g o 1-\Delta d b$ (AMy27032), $p G A L-s g o 1-4 R$ (AMy27033), $p G A L-s g o 1-4 R-\triangle d b$ (AMy27034), siz1 $\Delta$

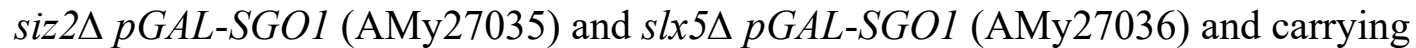
His-UBI (AMp1673) were arrested in G1 in raffinose medium and $p G A L-S G O 1$ $9 M Y C$ expression was induced by the addition of galactose. Ubiquitinated proteins 
were purified on Ni-NTA resin and Sgo1-9Myc was detected in inputs and elutes by anti-Myc immunoblot. (C) Sgo1 $\Delta d b$ and slx $5 \Delta$ do not noticeably change the levels of Sgo1-SUMO. In vivo SUMO assay was performed as described in Figure 2A. Cells analysed were wild type (AMy906), slx5s (AMy9765) and sgol- $\Delta d b$ (AMy18153), carrying Sgo1-6HA. (D-G) Sgo1 dissociation from the pericentromeres is important for promoting anaphase onset, but occurs independently of SUMOylation.

(D and E) Tethering Sgo1 to the kinetochore component Mtw1 blocks anaphase onset.

(D) Scheme of the experiment. Live cell imaging was performed in a/a diploid synchronised by release from G1. Metaphase duration was determined as the time between the observation of two Spc42-tdTomato dots until they reached a distance of $>2 \mu \mathrm{m}$ apart. (E) Metaphase duration was measured in at least $200 \mathrm{pGAL-SGO1-GBP}$ (AMy26679), MTW1-GFP (AMy26682), $p G A L-S G O 1-G B P M T W 1-G F P$ (AMy26568) and $p G A L-s g o 1-3 A-G B P M T W 1-G F P$ (AMy26570) cells carrying SPC42-tdTOMATO. (F) SUMOylation is not required for Sgol dissociation from the pericentromeres under tension. No tag control (AMy2508), SGO1-6HA (AMy6390) and siz1 1 siz2 $\triangle$ SGO1-6HA (AMy8115) cells carrying MET-CDC20 were arrested in metaphase in the presence (DMSO) or absence (benomyl/nocodazole) of spindle tension and Sgol association with the indicated site was measured by ChIP-qPCR. (G) SUMO-deficient Sgo1 mutants show similar localization to wild type Sgo1. Sgo1 association with CEN4 was measured by ChIP-qPCR using wild type (AMy25141), sgo1-2R (AMy26707), sgo1-4R (AMy26696) and sgo1-5R (AMy26708) strains carrying SGO1-6HIS-3FLAG, together with a no tag control (AMy2508). Cells were arrested in metaphase by depletion of $\mathrm{Cdc} 20$ in the presence or absence of spindle tension. $(\mathrm{H}) \operatorname{siz} 1 \Delta$ siz2 $\Delta$ delay in metaphase after bulk Sgol removal from the pericentromere. Wild type (AMy9233) and siz1 $\operatorname{siz} 2 \Delta$ (AMy15604) strains carrying MET-CDC20, SGO1-yeGFP and MTW1-dtTOMATO were followed by live cell imaging and the time elapsed between the indicated events was quantified for at least 50 cells. Anaphase onset was estimated as the time when the two Mtw1-tdTOMATO dots were $>2 \mu \mathrm{m}$ apart. 



Figure 5. SUMOylation of Sgol facilitates stable microtubulekinetochore attachments. (A and B) UnSUMOylated Sgol is proficient for sister kinetochore biorientation. (A) Experimental scheme for evaluating sister kinetochore biorientation after nocodazole washout. Briefly, cells were arrested in metaphase in the presence of nocodazole. After drug washout, cells remained arrested in metaphase and were fixed and visualized as described in 'Methods'. (B) In contrast to the sgol-3A mutant, sgol-2R, $-4 R$ and $\operatorname{siz} 1 \Delta$ siz $2 \Delta$ cells are proficient in the initial establishment of sister kinetochore biorientation, though $s g o 1-5 R$ is partially defective in biorientation. Strains used carried $p M E T-C D C 20$, CEN4-GFP and SPC42dtTOMATO, and were wild type (AMy4643), sgol-3A (AMy8923),

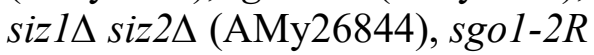
(AMy26753), sgo1-4R (AMy26278) and sgol-5R (AMy26758). The number of cells with two CEN4-GFP foci was determined for 200 cells. (C-E) Unstable kinetochore-microtubule attachments in SUMOylation mutants. (C) Scheme describing biorientation assay after nocodazole washout. All strains were imaged for a total of $3 \mathrm{~h}$ post

nocodazole washout. (D) Biorientation is unstable in siz $1 \Delta$ siz2 $\Delta$ and sgol-4R cells. Shown are the percentages of cells with the indicated number of switching(s) from

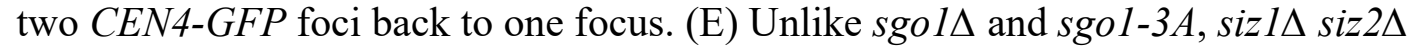
and sgol-4R cells do not show increased mis-segregation of chromosomes. The inheritance of $C E N 4-G F P$ by the daughter cells was analyzed for each strain. Strains used in (C-E) contained CEN4-GFP, pMET-CDC20 and SPC42-tdTOMATO and

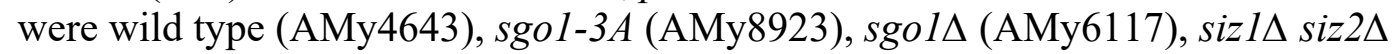
(AMy26844) and sgol-4R (AMy26278). Typically $>100$ cells and at least 50 cells were analyzed for each strain. at each time point. Error bars represent standard error of 3 biological replicates. 
A
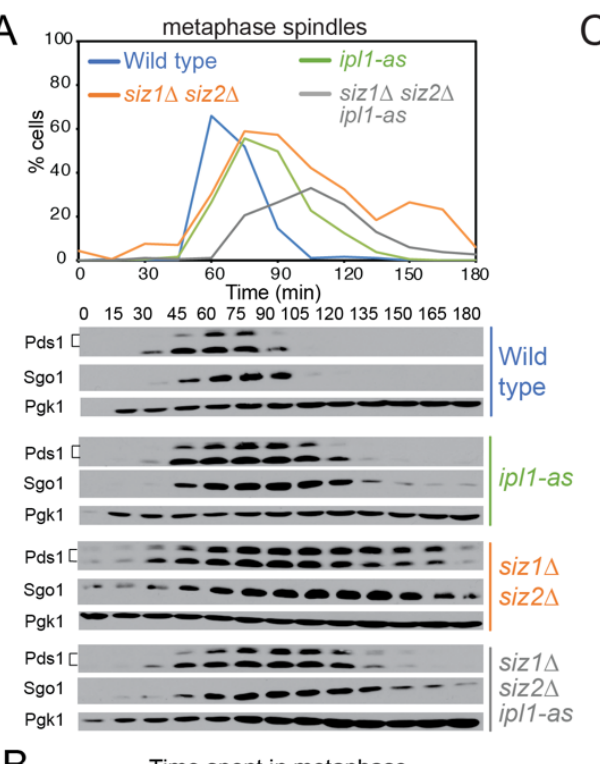

B

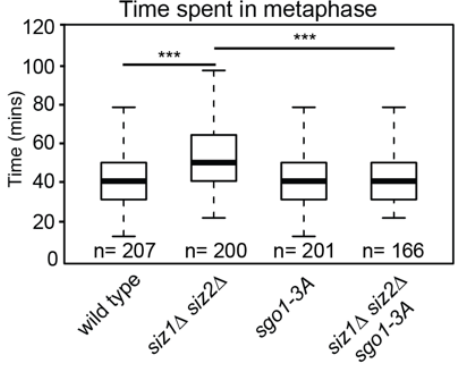

C Kinetochore localized- Ipl1 during the metaphase-anaphase transition
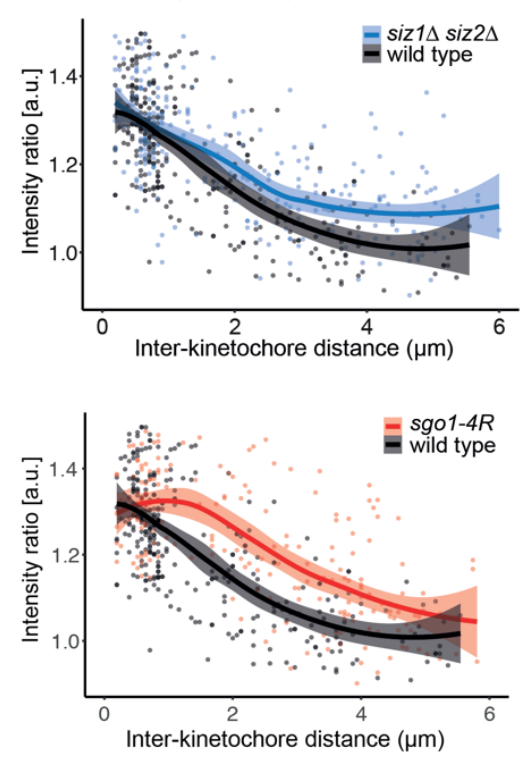

Figure 6. Sgo1 SUMOylation promotes timely removal of Ipl1 from kinetochores. (A) Inhibition of Ipl1 kinase activity partially rescues the metaphase delay phenotype of $\operatorname{siz} 1 \Delta$ siz $2 \Delta$ mutant. Cells were released from G1 arrest, NA-PP1 was added when small buds emerged, and cell cycle progression was assessed as described in Figure

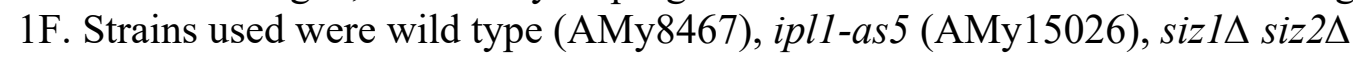

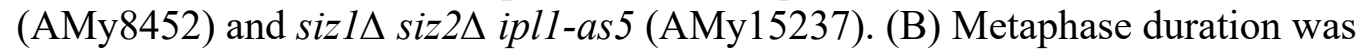
determined by live cell imaging as described in Figure 1D. Strains used carried TUB1-YFP and CDC14-GFP and were wild type (AMy24174), siz1D siz2A (AMy24313), sgol-3A (AMy24433) and sizl $\Delta$ siz2 $\Delta$ sgol-3A (AMy24471). $\mathrm{n}=$ number of cells analyzed. Data were presented as box plots, with the median values indicated by the thick black lines, upper and lower quartiles marked by the upper and lower borders of the boxes, and maximum (excluding outliers) and minimum values marked by the error bars. $P$-values were calculated using the Mann-Whitney U-test. $* * *=P<0.001$. (C) Ipl1 stays longer on kinetochores during the metaphaseanaphase transition in Sgo1 SUMO-deficient mutants. Strains used contained IPL1yeGFP, MET-CDC20 and MTW1-tdTOMATO, and were wild type (AMy9231), siz1 siz2 $\Delta$ (AMy15602) and sgo1-4R (AMy24143). Cells were released from G1 and were imaged on a microfluidics device. Line scans were performed across kinetochore foci of single cells, which measured the distance between the two Mtw1-tdTOMATO foci, as well as the Ipl1-GFP intensities co-localizing with the Mtw1 foci. Intensity ratio = the average intensity of the two Ipl1-GFP signals/average intensity of the two Mtw1tdTOMATO signals. Shaded areas represent 95\% confidence intervals. $>200$ line scans were performed for each strain. 
A


D

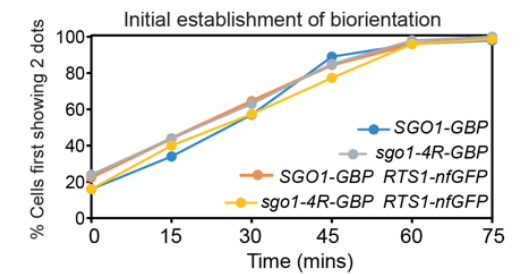

G

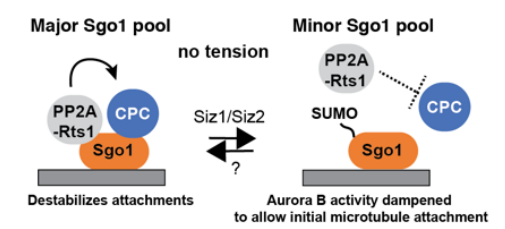

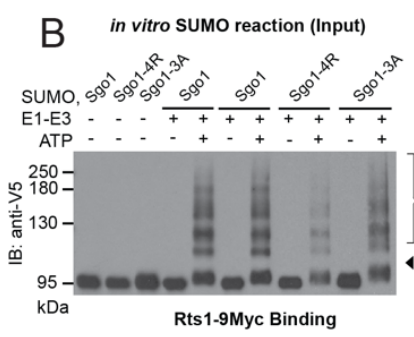
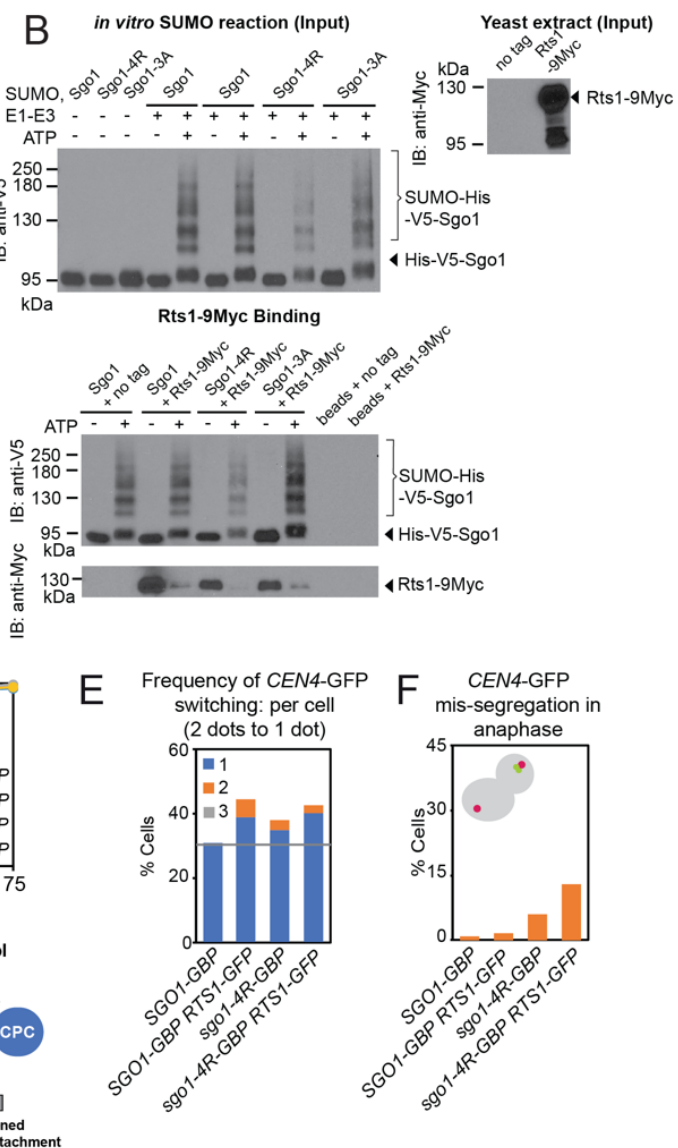

Figure 7. SUMOylation blocks Rts1 binding to Sgol and release of this interaction is important for stable biorientation. (A) The Sgo1-3A mutant which fails to associate with PP2A-Rts1 or CPC shows enhanced SUMOylation. In vivo SUMOylation assay was performed on wild type (AMy7655) and sgo1-3A (AMy25988) strains carrying SGO1-6HA. (B) SUMOylated Sgo1 has reduced binding affinity for Rts1.

Recombinant V5-tagged Sgo1 was mixed with components of the SUMOylation pathway in the presence or absence of ATP. Anti-V5 antibody coupled Protein G dynabeads were added to the mixture, washed thoroughly and incubated with extract from sgo1D or sgo1D RTS1-9MYC (AMy8832). Levels of Sgo1 and Rts1 bound to beads were probed by anti-V5 and anti-Myc western blotting, respectively. (C) Rts1 preferentially binds to unsumoylated Sgo1. Rts1-9MYC was immunoprecipitated from sgol二 RTS1-9MYC (AMy8832) using anti-MYC antibody coupled to Protein G dynabeads. Beads were incubated with in vitro SUMOylation reaction mixture containing Sgo1. Levels of Sgo1 and Rts 1 bound to beads were probed by anti-V5 and anti-Myc western blotting, respectively. (D-F) Biorientation is unstable when Rts 1 is tethered to wild type Sgo1 or Sgo1-4R. Strains used contained CEN4mNeonGreen MET-CDC20 and SPC42-tdTOMATO and were SGO1-GBP (AMy28389), SGO1-GBP RTS1-non-fluorescent GFP (AMy28092), sgo1-4R-GBP (AMy28417) and sgo1-4R-GBP RTS1-non-fluorescent GFP (AMy28416). The assay was performed as described in Figure 5C. (D) Tethering Rts 1 to wild type Sgol or Sgo1-4R does not affect the initial establishment of biorientation. (E) Increased reassociation of CEN4-mNeonGreen dots was observed when Rts1 was tethered to wild type Sgo1 or Sgo1-4R. (F) Mis-segregation is only modestly increased when Rts1 is tethered to wild type Sgo1 or Sgo1-4R. (G) Schematic model of how Sgo1 SUMOylation may alter the kinase-phosphatase balance to initiate error correction silencing and promote anaphase onset. For details, see text 


\section{Methods}

\section{$\underline{\text { Yeast strains and plasmids }}$}

Yeast strains are derivatives of W303 and are listed in Supplementary Table S3.

Plasmids and primers are listed in Supplementary Tables S4 and S5, respectively.

StuI-digested AMp1239 was transformed into a CDC14-GFP strain to make the YFPTUB1 CDC14-GFP parent strain. Genes were deleted or tagged using PCR-based transformation. K-R mutant plasmids were generated using Quikchange II XL sitedirected mutagenesis kit (Agilent), with primers listed in Table S5. K-R mutants were PCR amplified from the resulting plasmid using primers AM16 and AM3177 and were integrated into an sgols strain (AMy827). 7HIS-SMT3 and HIS-UBI plasmids were kind gifts from Dr. H. Ulrich.

\section{$\underline{\text { Yeast growth and synchronization }}$}

Unless otherwise stated, yeast strains were grown in YEP supplemented with $2 \%$ glucose and $0.3 \mathrm{mM}$ adenine (YPDA). For the benomyl sensitivity assays, plates were made by adding $10 \mu \mathrm{g} / \mathrm{mL}$ benomyl (Sigma) or the equivalent volume of DMSO (solvent control) to boiling media.

To synchronize cells in G1, overnight cultures were inoculated to $\mathrm{OD}_{600}=0.2$ and grown for $1 \mathrm{~h}$ at room temperature, before diluting back to $\mathrm{OD}_{600}=0.2$. $\alpha$-factor was added to $5 \mu \mathrm{g} / \mathrm{mL}$ for $90 \mathrm{~min}$ and then re-added to $2.5 \mu \mathrm{g} / \mathrm{mL}$ for every $90 \mathrm{~min}$, until > 95\% cells exhibited shmooing morphology. To release cells from G1, $\alpha$-factor was washed out using $10 \times$ volume relevant media. For $p M E T-C D C 20$-containing strains, cells were arrested in G1 in methionine dropout medium. After $\alpha$-factor wash-out, cells were released into YPDA (+ DMSO or $+30 \mu \mathrm{g} / \mathrm{mL}$ benomyl and $15 \mu \mathrm{g} / \mathrm{mL}$ nocodazole) $+8 \mathrm{mM}$ methionine for $1 \mathrm{~h} .4 \mathrm{mM}$ methionine and DMSO/15 $\mu \mathrm{g} / \mathrm{mL}$ nocodazole were re-added for $1 \mathrm{~h}$.

\section{Metaphase duration measurements by live cell imaging and mitotic time course}

Synthetic complete/dropout media were used for growing and washing cells for live cell imaging. Cells released from G1-arrest were loaded onto $\mu$-slide dishes (Ibidi) coated with concanavalin-A (Sigma). Images were taken at indicated time intervals using a Zeiss inverted microscope, in a temperature-controlled chamber $\left(25^{\circ} \mathrm{C}\right.$ for 
glucose-based media and $30^{\circ} \mathrm{C}$ for raffinose-based media). Movies were analyzed using the ImageJ software.

Mitotic time course experiments were performed at $25^{\circ} \mathrm{C}$. To inhibit Ipl1-as5, NAPP1 (Toronto Research Chemicals) was added to a final concentration of $50 \mu \mathrm{M}$ upon the emergence of small-budded cells. For every time point, samples were either treated with 5\% trichloroacetic acid (TCA) for protein extraction, or fixed in $3.7 \%$ formaldehyde for immunofluorescence. TCA-treated pellets were snap frozen in liquid nitrogen, washed in acetone and air-dried. Protein samples were prepared by bead beating and boiling in SDS sample buffer. For immunofluorescence, Fixed cells were spheroplasted using zymolyase (AMS Biotechnology) and glusulase (Perkin Elmer), fixed in methanol for $3 \mathrm{~min}$, followed by $10 \mathrm{~s}$ incubation in acetone. Rat anti$\alpha$-tubulin (Abd Serotec) antibody was used at 1:50 and anti-Rat FITC (Jackson ImmunoResearch) was used at 1:16.7. Spindle morphology of 200 cells was analyzed for each time point.

\section{Western blotting}

Proteins were separated in $8 \%$ bis-tris acrylamide gels and were transferred to nitrocellulose membranes (except anti-ubiquitination blots, for which PVDF membranes were used). Membranes were blocked in 3-5\% milk in phosphatebuffered saline $+0.1 \%$ Tween-20 (PBST) or tris-buffered saline $+0.1 \%$ Tween-20 (TBST). All antibodies were diluted in 2\% milk PBST (except anti-ubiquitination blot, for which 2\% milk TBST was used). Anti-c-Myc (9E10, Biolegend), anti-V5 (AbD Serotec), anti-Flag (M2, Sigma) and anti-HA (12CA5, Roche) antibodies were used at 1:1000 dilution. Anti-Pgk1 (lab stock) and anti-Kar2 (lab stock) loading controls were used at 1:10000 and 1:100,000 dilution, respectively. The nonquantitative blots were detected by ECL (Thermofisher) and autoradiograms. 20\% Femto-ECL (Thermofisher) diluted in Pico-ECL was used to detect SUMOylatedSgo1 signals. Quantitative blots were detected using a BioRad Chemidoc (Figure S4C) or a LiCOR Odyssey Clx machine (Figure S4D). Protein expression was quantified using ImageJ (Figure S4C) or ImageStudio (Figure S4D). 
Cultures were inoculated to $\mathrm{OD}_{600}=0.2$ in $200 \mathrm{~mL}$ synthetic dropout media and grown for $4 \mathrm{~h}$ at room temperature. Equal OD of cells were collected for samples in the same experiment. Cell pellets were resuspended in $20 \mathrm{~mL}$ cold $\mathrm{H}_{2} \mathrm{O}$ and incubated with $3.2 \mathrm{~mL}$ solution containing $1.85 \mathrm{M}$ sodium hydroxide and $7.5 \% \beta$ mercaptoethanol. After $20 \mathrm{~min}$ incubation on ice, $1.65 \mathrm{~mL} \mathrm{100 \%} \mathrm{trichloroacetic} \mathrm{acid}$ was added and cells were incubated on ice for a further $20 \mathrm{~min}$. Cell pellets were drop-frozen in liquid nitrogen, and subsequently lysed by bead-beating in $300 \mu \mathrm{L}$ buffer A (6 M guanidine hydrochloride, $100 \mathrm{mM}$ sodium phosphate buffer $\mathrm{pH}$ 8.0, 10 $\mathrm{mM}$ Tris- $\mathrm{HCl} \mathrm{pH}$ 8.0). Lysate was diluted three-fold in buffer $\mathrm{A}$ and $10 \mu \mathrm{L}$ was saved as input controls. Lysate was applied to a column packed with $600 \mu \mathrm{L} 50 \%$ slurry NiNTA agarose beads (Qiagen), washed twice with buffer A $+0.05 \%$ Tween-20, twice with buffer B (8 M urea, $100 \mathrm{mM}$ sodium phosphate buffer $\mathrm{pH} 6.8,10 \mathrm{mM}$ Tris- $\mathrm{HCl}$ $\mathrm{pH} 6.8)+0.05 \%$ Tween-20 and once with buffer B $+0.05 \%$ Tween- $20+20 \mathrm{mM}$ imidazole. SUMOylated proteins were eluted by buffer B $+0.05 \%$ Tween-20 +200 $\mathrm{mM}$ imidazole. Input and elute samples were concentrated by centrifugation using Vivaspin columns (Sartorius).

\section{Mass spectrometry}

A $p G A L-S G O 1$ strain with $7 H I S-S M T 3$ was inoculated to 0.2 OD in $2 \%$ raffinose media. After $3 \mathrm{~h}$ growth at room temperature, cells were diluted back to $0.2 \mathrm{OD}$ and $2 \%$ galactose was added to induce $S G O 1$ overexpression. Cells were harvested after 3 $\mathrm{h}$ and SUMOylated proteins were purified as described above. Proteins eluted from the Ni-NTA column were separated on a 4-12\% NuPage Bis-Tris gel (Invitrogen) and the gel slice encompassing SUMOylated Sgol (between $\sim 100 \mathrm{kDa}$ and $135 \mathrm{kDa}$, based on immunoblotting of a parallel gel) was excised for mass spectrometry analysis.

\section{Cloning, expression and purification of recombinant Sgol}

Full-length wild type $S G O 1$ was amplified from plasmid AMp899, to replace $S M T 3$ in plasmid AMp773 by Gibson assembly using primers AM8849 to AM8852. V5 tag was inserted by Gibson assembly using plasmid AMp970 and primers AM8866 to AM8869 to generate N-terminal tobacco etch virus (TEV) protease cleavable $\mathrm{His}_{\mathrm{x} 7-}$ V5 tag-tagged SGO1 (AMp1738) under the control of a $p C U P 1$ promoter. sgol-4R 
was PCR amplified from plasmid AMp1340 and ligated into AMp1738 by Gibson assembly using primers AM8850, AM8851, AM9124 and AM 9125 to generate Nterminal TEV protease cleavable $\mathrm{His}_{\mathrm{x}} \mathrm{7}$-V5 tag-tagged sgol-4R (AMp1783).

A protease-deficient yeast strain (AMy8184) was transformed with the resulting plasmids (AMp1738 or AMp1783) and the transformants were inoculated into $8 \mathrm{~L}$ uracil dropout media. When $\mathrm{OD}_{600}$ reached $0.5-0.7,0.5 \mathrm{mM} \mathrm{CuSO}_{4}$ was added to induce $\mathrm{His}_{\mathrm{x} 7-\mathrm{V} 5}$-SGO1 (or sgol-4R) expression. Cells were harvested $6 \mathrm{~h}$ after induction. Cell pellets were snap frozen in liquid nitrogen and ground to powder in a ball breaker machine (Retsch). All purification steps were performed at $4^{\circ} \mathrm{C}$ or on ice. Cell powder was resuspended in lysis buffer ( $25 \mathrm{mM}$ Tris- $\mathrm{HCl} \mathrm{pH} 7.5,150 \mathrm{mM} \mathrm{NaCl}$, $1 \mathrm{mM} \mathrm{MgCl}_{2}, 10 \%$ glycerol, 0.1\% NP-40, 0.05 mM EDTA, 0.05 mM EGTA, 1 mM DTT, 1xCLAAPE, $1 \mathrm{mM}$ Pefabloc, $0.4 \mathrm{mM}$ Na Orthovanadate, $0.1 \mathrm{mM}$ Microcystin, $1 \mathrm{mM}$ NEM, $2 \mathrm{mM}$ B-glycerophosphate, $1 \mathrm{mM}$ Na pyrophosphate, $5 \mathrm{mM} \mathrm{NaF}$, complete EDTA-free protease inhibitor (Roche)). Cell lysates were treated with 40 $\mathrm{u} / \mathrm{mL}$ benzonase for $1.5 \mathrm{~h}$. The crude lysate was diluted with $25 \mathrm{mM}$ sodium phosphate buffer $\mathrm{pH} 7.5,500 \mathrm{mM} \mathrm{NaCl}, 10 \%$ glycerol, $0.1 \% \mathrm{NP}-40,10 \mathrm{mM}$ imidazole, $1 \mathrm{mM}$ DTT, $0.25 \mathrm{mM}$ PMSF, cleared by ultracentrifugation $(50,000 \times \mathrm{g}$ at $4^{\circ} \mathrm{C}$ for $30 \mathrm{~min}$ ), filtered through a $0.22 \mu \mathrm{m}$ filter and loaded onto a HiTrap IMAC FF $1 \mathrm{ml}$ column (GE Healthcare) charged with $\mathrm{Ni}^{2+}$ and attached to an AKTA system. The column was washed with $25 \mathrm{mM}$ sodium phosphate buffer $\mathrm{pH} 7.5,500 \mathrm{mM}$ $\mathrm{NaCl}, 10 \%$ glycerol, 0.1\% NP-40, $25 \mathrm{mM}$ imidazole, $1 \mathrm{mM}$ DTT, $0.25 \mathrm{mM}$ PMSF. Recombinant Sgol protein was eluted with an increasing imidazole gradient (25- 500 $\mathrm{mM}$ ) over 40 column volumes and then loaded onto a gel filtration Superose 6 10/300 column in $50 \mathrm{mM}$ Tris- $\mathrm{HCl} \mathrm{pH}$ 7.5, $500 \mathrm{mM} \mathrm{NaCl}, 10 \%$ glycerol, $0.1 \mathrm{mM}$ EDTA, 0.1 mM EGTA, $1 \mathrm{mM}$ DTT, $0.25 \mathrm{mM}$ PMSF. Fractions containing the eluted protein were run on an SDS-PAGE gel and the purity of recombinant Sgol was assessed by InstantBlue (Expedeon) staining.

\section{Cloning, expression and purification of of SUMOylation enzymes}

SUMO E1 enzymes Uba2 and Aos1 (from vectors pET11a-UBA2 and pET28a-AOS1, [53]) were co-expressed in BL21 CodonPlus (DE3) RIL (Agilent Technologies) in the presence of $0.05 \mathrm{mM} \mathrm{IPTG}$ at $18{ }^{\circ} \mathrm{C}$ for $20 \mathrm{~h}$. Expression of SUMO E2 enzyme Ubc9 and SUMO Smt3 (pET21b-UBC9 and pET21a-SMT3 [53]) was induced by $0.1 \mathrm{mM}$ 
IPTG at $18{ }^{\circ} \mathrm{C}$ for $20 \mathrm{~h}$ in BL21 CodonPlus (DE3) RIL. E1, E2 and SUMO were purified using Ni-NTA agarose resin (Qiagen) with $20 \mathrm{mM}$ sodium phosphate $\mathrm{pH} 7.5$, $500 \mathrm{mM} \mathrm{NaCl}, 10$ to $200 \mathrm{mM}$ imidazole, followed by Superdex 200 16/600 column (hand poured using prep grade resin from GE Healthcare) in $50 \mathrm{mM}$ Tris- $\mathrm{HCl} \mathrm{pH} 7.5$, $150 \mathrm{mM} \mathrm{NaCl}, 1 \mathrm{mM}$ DTT, $10 \%$ glycerol.

siz1-167-508 and siz2-130-490 were amplified from plasmids pGEX-4T-1-SIZ1-HF [53]) and AMp1528 (YEPlac112-SIZ2, this work), respectively. The respective PCR product was cloned into pMAL-c2X to generate N-terminal MBP-tagged siz1-167508 (AMp1679) and N-terminal MBP-tagged siz2-130-490 (AMp1680). Siz1 (167508) or Siz2 (130-490) were expressed in LB containing $10 \mu \mathrm{M} \mathrm{ZnCl}_{2}, 0.1 \mathrm{mM}$ IPTG and appropriate antibiotics at $16{ }^{\circ} \mathrm{C}$ for $20 \mathrm{~h}$ using BL21 CodonPlus (DE3) RIL (Agilent Technologies). Siz1 (167-508) or Siz2 (130-490) was purified from MBPTrap HP $1 \mathrm{~mL}$ column (GE Healthcare) with $50 \mathrm{mM}$ Tris-HCl pH 7.5, $150 \mathrm{mM}$ $\mathrm{NaCl}, 10 \mu \mathrm{M} \mathrm{ZnCl}_{2}, 0$ to $10 \mathrm{mM}$ maltose gradient, followed by gel filtration with a Superdex 200 16/600 column (hand poured using prep grade resin from GE Healthcare) in $50 \mathrm{mM}$ Tris- $\mathrm{HCl} \mathrm{pH} 7.5,150 \mathrm{mM} \mathrm{NaCl}, 10 \mu \mathrm{M} \mathrm{ZnCl}_{2}, 10 \%$ glycerol.

\section{$\underline{\text { In vitro SUMOylation assays and SUMOylated Sgol pull down assays }}$}

Purified Sgol $(1 \mu \mathrm{M})$ was mixed with $5 \mathrm{mM}$ ATP, $15 \mu \mathrm{M}$ SUMO, $0.5 \mu \mathrm{M}$ E1 (unless otherwise indicated), $0.5 \mu \mathrm{M}$ E2 (unless otherwise indicated), $0.1 \mu \mathrm{M}$ (unless otherwise indicated) Siz1 (167-508) or Siz2 in a reaction buffer consisting of $25 \mathrm{mM}$ HEPES pH 7.5, $150 \mathrm{mM} \mathrm{KCl,} 10 \mathrm{mM} \mathrm{MgCl}$, 15 \% glycerol, $0.1 \%$ NP-40, $0.1 \mathrm{mM}$ DTT, $0.25 \mathrm{mM}$ PMSF. The reaction was incubated at $30^{\circ} \mathrm{C}$ for $2 \mathrm{~h}$ (unless otherwise indicated). For detection of SUMOylated Sgo1, the reaction was boiled in SDS sample buffer before analyzed by anti-V5 western blotting.

For the binding assay, product of in vitro SUMOylation was incubated with $50 \mu \mathrm{L}$ of anti-V5 coupled protein G magnetic dynabeads (Thermofisher) in Binding buffer A (25 mM HEPES pH 7.5, 150 mM KCl, 2 mM MgCl $2,15 \%$ glycerol, 0.1\% NP-40, 0.1 mM EDTA, $0.5 \mathrm{mM}$ EGTA, $0.25 \mathrm{mM}$ PMSF) for $2.5 \mathrm{~h}$ at $4{ }^{\circ} \mathrm{C}$. After washing three times with Binding buffer A, beads were incubated with yeast lysate containing 
tagged Rts1 or Bir1 for $2 \mathrm{~h}$ at $4{ }^{\circ} \mathrm{C}$. Beads were washed five times with binding buffer $\mathrm{A}+10 \mathrm{mM}$ NEM and were heated at $65^{\circ} \mathrm{C}$ for $15 \mathrm{~min}$ to elute bound proteins.

\section{Analysis of in vivo ubiquitination}

Cultures were inoculated to $\mathrm{OD}_{600}=0.2$ in $100 \mathrm{~mL} 2 \%$ raffinose-containing dropout media and were arrested in G1 as described above. $2 \%$ galactose was added for 30 min to induce $p G A L-S G O 1-9 M Y C$ expression. Cells were treated, lysed in buffer A and diluted as described in in vivo SUMOylation assay. Lysates were incubated with Ni-NTA beads, $0.05 \%$ Tween-20 and $15 \mathrm{mM}$ imidazole overnight at $4^{\circ} \mathrm{C}$, with gentle rotation. Beads were washed twice with buffer $A+0.05 \%$ Tween-20, and four times with buffer C (8M urea, $100 \mathrm{mM}$ sodium phosphate buffer $\mathrm{pH} 6.3,10 \mathrm{mM}$ Tris-HCl, $\mathrm{pH} 6.3)+0.05 \%$ Tween-20. For elution, beads were heated at $60^{\circ} \mathrm{C}$ for $10 \mathrm{~min}$ in $\mathrm{HU}$ buffer (8M urea, $200 \mathrm{mM}$ Tris-HCl pH 6.8, 1 mM EDTA, 5\% (w/v) SDS, 0.1\% (w/v) bromophenol blue, $1.5 \%(\mathrm{w} / \mathrm{v}) \mathrm{DTT})$.

\section{$\underline{\text { Sgo1 half-life measurement }}$}

Cultures were inoculated to $\mathrm{OD}_{600}=0.2$ in YEP $+2 \%$ raffinose $+0.3 \mathrm{mM}$ adenine. 5 $\mu \mathrm{g} / \mathrm{mL}$ alpha-factor was added for $1.5 \mathrm{~h}$ and re-added to $2.5 \mu \mathrm{g} / \mathrm{mL}$ every hour until the end of the experiment. SGO1 overexpression was induced by the addition of $2 \%$ galactose for $30 \mathrm{~min}$. De novo synthesis of Sgol protein was quenched by the addition of $2 \%$ glucose and $1 \mathrm{mg} / \mathrm{mL}$ cycloheximide (Acros Organics).

\section{Co-immunoprecipitation}

Cultures were inoculated to $\mathrm{OD}_{600}=0.2$ in $2 \mathrm{~L}$ YPDA and grown at $30{ }^{\circ} \mathrm{C}$ until $\mathrm{OD}_{600}$ reached 1-1.5. Cell pellets were harvested by centrifugation and drop-frozen in liquid nitrogen. Cells were ground, lysed and benzonase-treated as described in 'Purification of recombinant protein'. After centrifugation at 3,600 rpm for $10 \mathrm{~min}$, protein concentrations were measured by Bradford assay and approximately $85 \mathrm{mg}$ of protein was incubated with $4 \mathrm{mg}$ of epoxy beads (Thermofisher) coupled to IgG for $1.5 \mathrm{~h}$ at $4^{\circ} \mathrm{C}$. Beads were washed four times with the lysis buffer and were heated at $70^{\circ} \mathrm{C}$ for $10 \mathrm{~min}$ in $50 \mu \mathrm{L} 1 \mathrm{x}$ LDS sample buffer $+2.5 \% \beta$-mercaptoethanol. $5 \mu \mathrm{L}$ of input and $20 \mu \mathrm{L}$ of IP samples were separated on an $8 \%$ acrylamide gel and were transferred to PVDF membranes. Western blot was performed using TBST-based buffer. 


\section{Chromatin immunoprecipitation (ChIP)}

Metaphase-arrested cells were fixed in $1.1 \%$ formaldehyde for at least $30 \mathrm{~min}$. Cells were washed twice with TBS (20 $\mathrm{mM}$ Tris- $\mathrm{HCl} \mathrm{pH} 7.5,150 \mathrm{mM} \mathrm{NaCl})$, once with FA lysis buffer (50 mM HEPES-KOH pH 7.5, 150 mM NaCl, 1 mM EDTA, 1\% Triton-X, $0.1 \%$ sodium deoxycholate) $+0.1 \%$ SDS, and drop frozen in liquid nitrogen. Cells were lysed by bead beating in FA lysis buffer $+0.5 \% \mathrm{SDS}+1 \mathrm{mM}$ PMSF + EDTA-free protease inhibitor cocktail (Roche). The resulting pellets were sonicated in a Bioruptor machine. Sheared chromatin was incubated with the relevant antibody and protein $\mathrm{G}$ dynabeads (Thermofisher) at $4{ }^{\circ} \mathrm{C}$ overnight. Anti-HA (12CA5, Roche Diagnostics), anti-Flag M2 (Sigma), or anti-V5 (Abd Serotec) antibodies were used as relevant. Beads were washed once with FA lysis buffer + $0.1 \% \mathrm{SDS}+275 \mathrm{mM} \mathrm{NaCl}$, once with FA lysis buffer $+0.1 \% \mathrm{SDS}+500 \mathrm{mM} \mathrm{NaCl}$, once with wash buffer (10 mM Tris-HCl pH 8, 0.25 M LiCl, 1 mM EDTA, 0.5\% NP$40,0.5 \%$ sodium deoxycholate) and once with TE (10 mM Tris-HCl, pH 8, $1 \mathrm{mM}$ EDTA). Chromatin was eluted by boiling in the presence of $10 \%$ Chelex beads, treated with proteinase $\mathrm{K}$ (Life technologies) for $30 \mathrm{~min}$ and boiling for a further 10 min. Quantitative PCR was done using Express SybrGreenER (Thermofisher) (CEN4 and ARM4) or Luna (NEB) (all other chromosomal sites) on a Lightcycler machine (Roche). Primers used were listed in Table S5. ChIP enrichment was determined using the formula $\mathrm{E}^{-\Delta \mathrm{Ct}}$, where $\Delta \mathrm{Ct}=\left(\mathrm{Ct}_{(\mathrm{ChIP})}-\left[\mathrm{Ct}_{\text {(input })}-\log \mathrm{E}\right.\right.$ (Input dilution factor $\left.)\right]$ and E represents primer efficiency.

\section{Biorientation assay in fixed and live cells}

Cells carrying pMET-CDC20, SPC42-tdTOMATO and CEN4-GFP were arrested in metaphase in the presence of nocodazole and benomyl as described in 'yeast growth and synchronization'.

For analyzing biorientation in metaphase-arrested cells (Figure 5C and 5D), drugs were washed out by filtering with 10 times the volume of YEP and cells were released into YPDA $+8 \mathrm{mM}$ methionine. Samples were taken at the indicated time points, fixed in $3.7 \%$ formaldehyde for 9 min, washed in $80 \%$ ethanol and resuspended in 1 
$\mu \mathrm{g} / \mathrm{mL}$ DAPI in PBS. 200 cells were analyzed for each time point using fluorescent microscope.

For analyzing biorientation in cells going into anaphase (Figure 5E and 7F), nocodazole-arrested cells were loaded onto the ONIX Microfluidic Perfusion System (CellAsic) and visualized with a Zeiss inverted microscope coupled to an EMCCD camera at $25^{\circ} \mathrm{C}$. Imaging started as soon as cells were released into methionine dropout media without drugs. 


\section{Supplementary information}

Table S1. Complete list of high copy suppressors of GAL-SGO1 sickness identified in the screen shown in Figure S1A.

Table S2. (A) List of SUMOylated peptides identified in mass spectrometry

(B) List of peptides identified in mass spectrometry

Table S3. Yeast strains used in this study

Table S4. Plasmids used in this study

Table S5. Oligonucleotides used in this study 
A
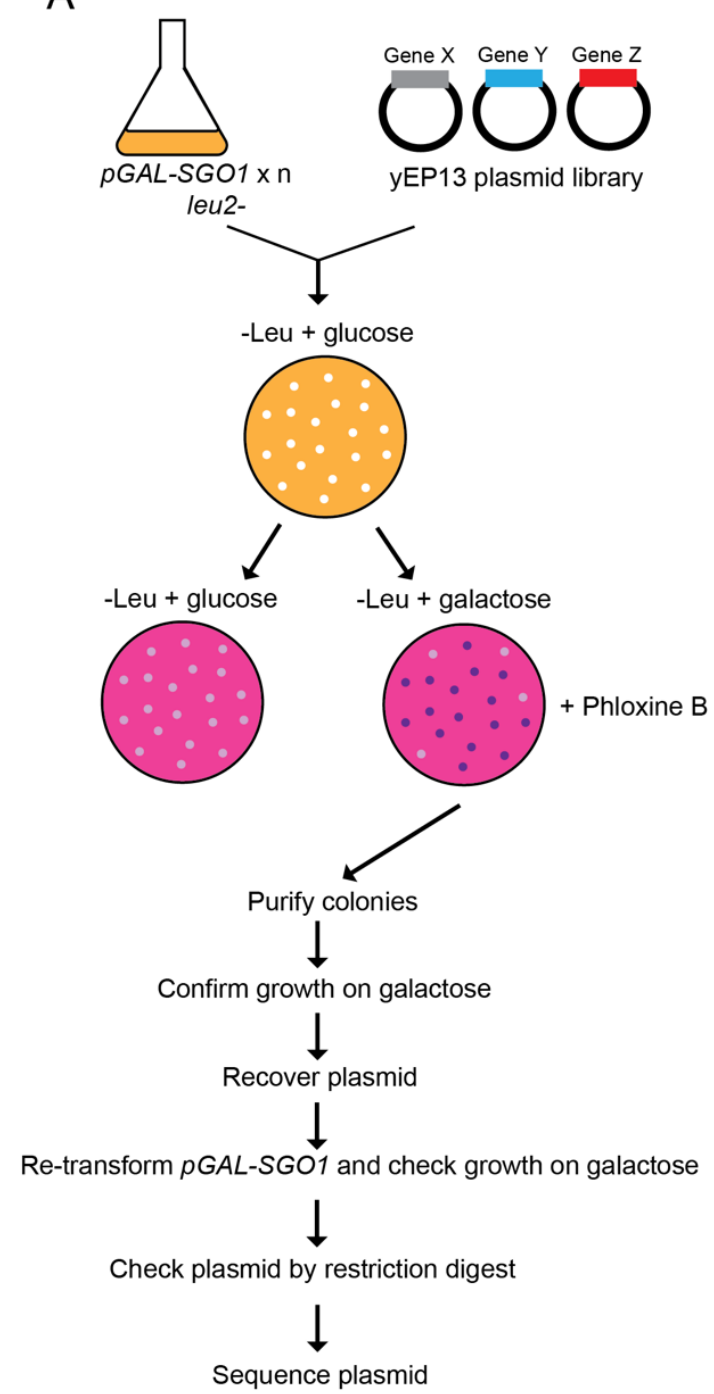

B
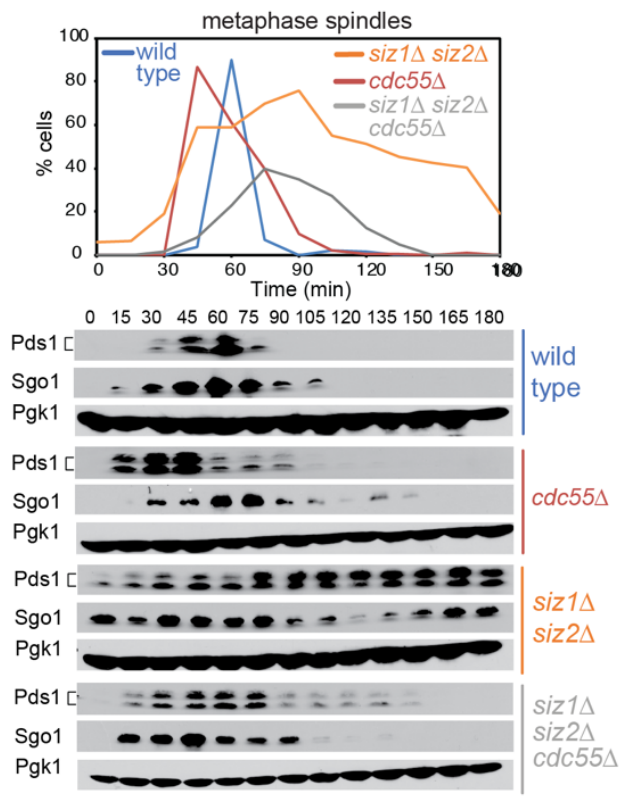

Figure S1, related to Figure 1. Identification of SUMO ligases as Sgo1 regulators. (A) Overview of multi-copy suppressor screen to identify Sgol antagonists. (B) Deletion of $C D C 55$ partially alleviates the metaphase delay phenotype of the sizl $1 \Delta$ siz $2 \Delta$ mutant. Mitotic time course analysis was performed as described in Figure 1F,

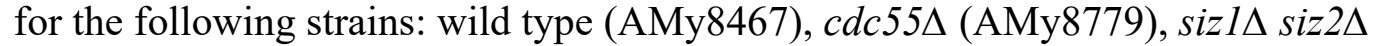

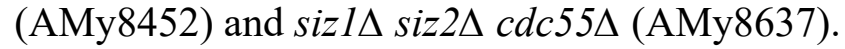






Figure S2, related to Figure 2. Purification of Sgo1. Coomassie staining confirmed the successful purification of wild type and mutant Sgol recombinant proteins. 

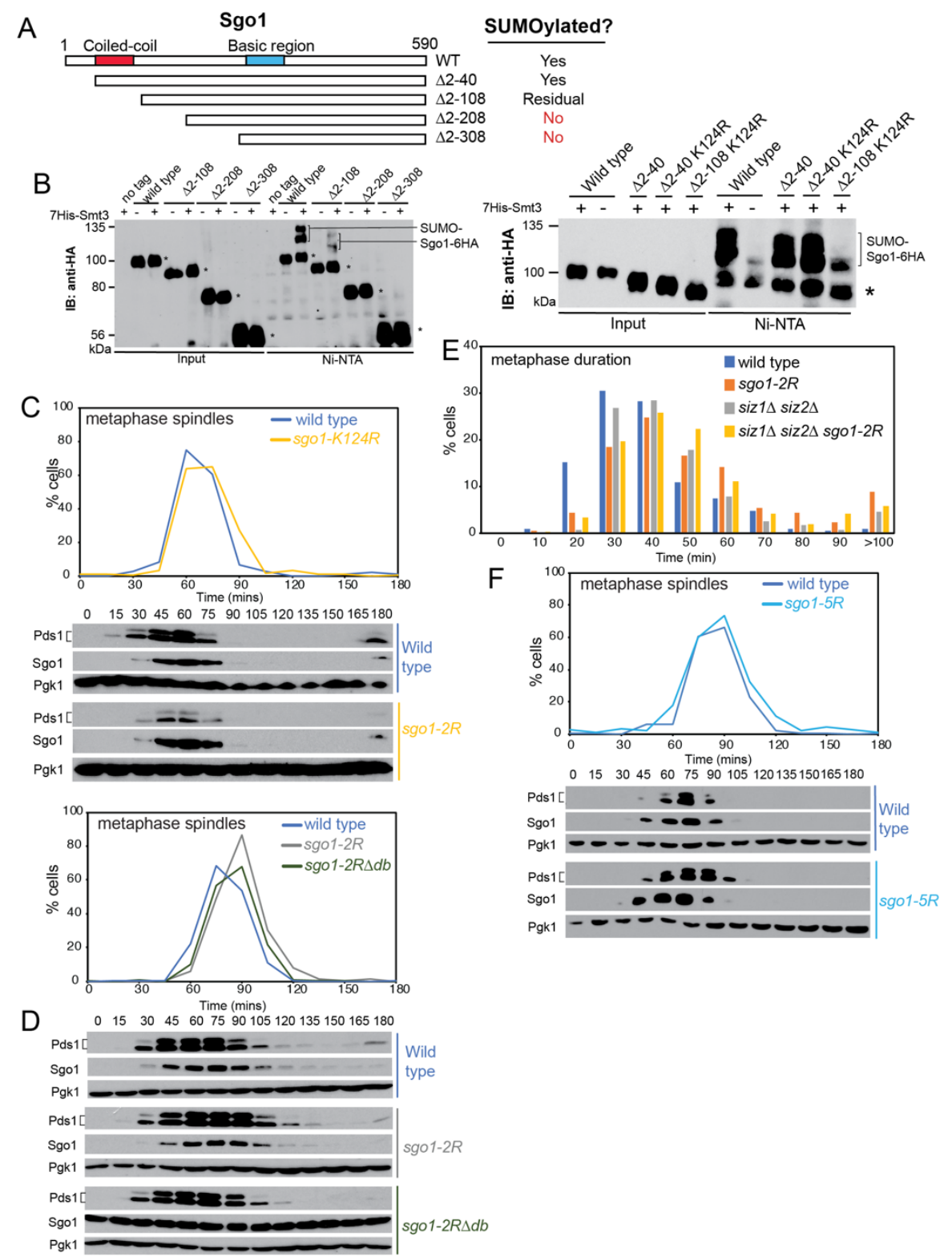

Figure S3, related to Figure 3. Identification and characterization of unSUMOylatable Sgo1 mutants. (A) Schematics describing the truncation mutants generated for Sgo1. The conserved coiled-coil and basic domains are highlighted in red and blue, respectively. Results from (B) are summarized on the right. (B) Sgol is likely to be SUMOylated in the first 208 amino acids. In vivo SUMOylation was assessed for the following Sgo1-6HA tagged strains as described in Figure 2A, together with the indicated negative controls: wild type (AMy7654), sgol $\Delta 2-108$

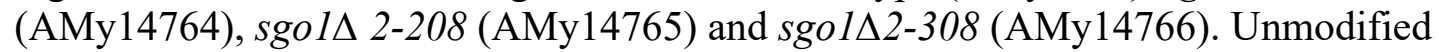
Sgol bands are marked with asterisks. In addition to Lys124 SUMOylation, identified by mass spectrometry, the region between amino acids 41 and 108 is likely to be SUMOylated. In vivo SUMOylation was assessed for the following Sgo1-6HA tagged strains: wild type (AMy7654), sgo1 $\Delta 2-40$ (AMy18194), sgol $\Delta 2-40$ K124R (AMy18476) and sgo1 $\Delta 2-108$ K124R (AMy16540). (C-F) Characterization of unSUMOylatable Sgo1 mutants. (C) The Sgo1-K124R mutants does not show a metaphase delay. Mitotic time course analysis as described in Figure $1 \mathrm{~F}$ was performed for wild type (AMy8467) and sgo 1-K124R (AMy24448) strains carrying 
SGO1-9MYC and PDS1-HA. (D) The sgo1-2R mutant shows a very minor metaphase delay that is not exacerbated by mutations in the Sgol destruction box. Analysis of sgo1-2R (AMy24448) and sgo1-2R- $\triangle d b$ (AMy25924) cells carrying SGO1-9MYC and $P D S 1-3 H A$ as described in Figure 1F. (E) Metaphase duration was measured after live cell imaging of wild type (AMy24546), siz1 $\operatorname{siz2\Delta }$ (AMy24547), sgo1-2R

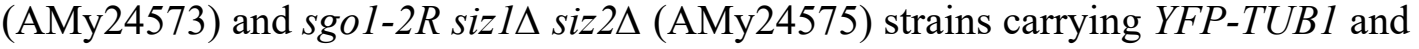
$C D C 14-G F P$ as described in Figure 1D. (F) sgol-5R (AMy24450) does not show a metaphase delay, suggesting adverse effects of K124R on Sgo1 protein function when combined with the $4 \mathrm{R}$ mutations. 
A
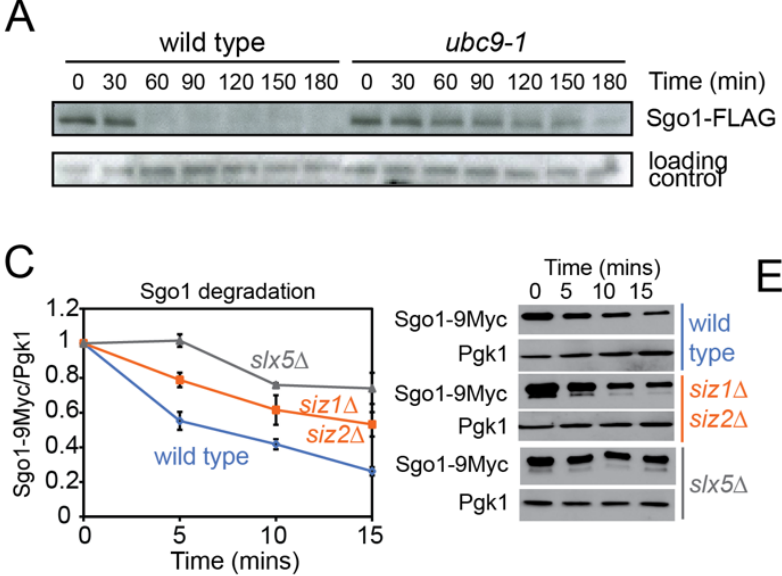

D
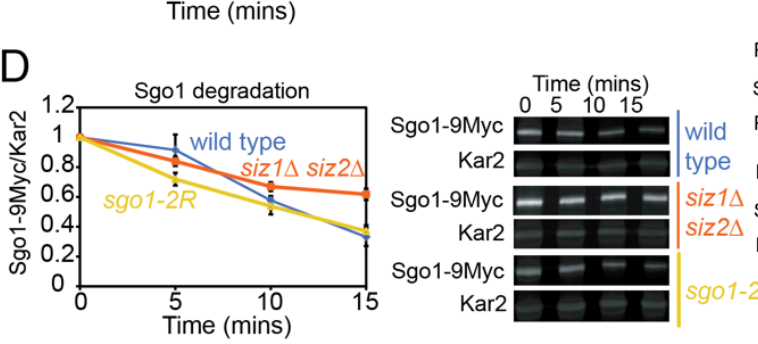

B

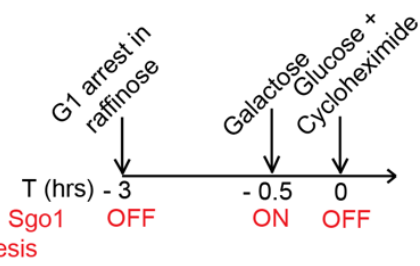

de novo Sgo
synthesis
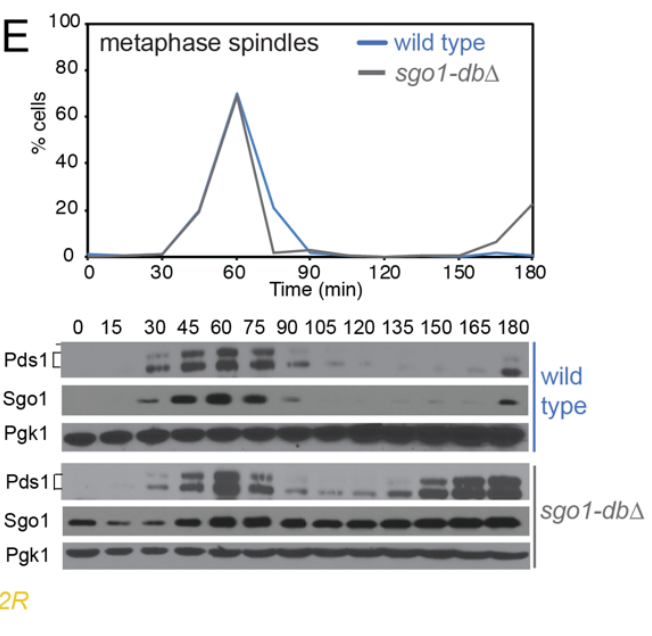


Figure S4, related to Figure 4. Analysis of factors promoting Sgol degradation. (A) The degradation of Sgol depends on SUMO-conjugating protein Ubc9. The cells were synchronized in nocodazole and released into medium with $\alpha$-factor to ensure arrest in G1. (B and C) Sgol half-life is increased in slx5 5 and siz1 $1 \Delta$ siz2 $\Delta$ mutants. (B) Scheme describing the cycloheximide chase experiment. Cells were arrested in G1 throughout the experiment and pGAL1-SGO1-9MYC expression was initially prevented by growth of cells in raffinose. Subsequently, a pulse of Sgol was provided by the addition of galactose, after which de novo Sgo1-9Myc synthesis was quenched 
by the addition of glucose (to block $p G A L$ expression) and cycloheximide (to block protein synthesis). (C) Cycloheximide chase experiment was performed for $p G A L$ -

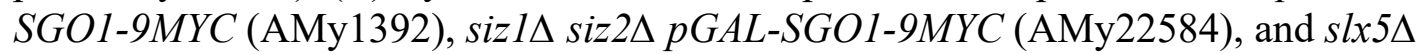
pGAL-SGO1-9MYC (AMy22586) strains. Representative anti-Myc immunoblot (right) to detect Sgo1-9Myc is shown. Pgk1 is shown as a loading control. Quantification showing the mean values from three biological replicates (left) is also shown. Relative Sgo1 levels were calculated as the ratio of Myc signal to Pgk1 signal and the ratio was set to 1 for time 0 . Error bars represent standard error calculated from three independent experiments. (D) The effects of SUMOylation on Sgo1 halflife are likely to be indirect. Sgol degradation is unaffected in the sgol-2R mutants. Cycloheximide chase experiment was performed for $p G A L-S G O 1-9 M Y C$ (AMy1392), siz1 $\operatorname{siz} 2 \Delta$ pGAL-SGO1-9MYC (AMy22584) and $p G A L-s g o 1-2 R-9 M Y C$ (AMy24940) strains. Kar2 was probed as a loading control. Quantification and statistics were performed as described in (C). (E) Sgo1 degradation is not required for timely anaphase entry. Time course analysis of wild type (AMy18500) and sgol- $\Delta \mathrm{db}$ (AMy18440) strains carrying SGO1-6HA and PDS1-18MYC as described in Figure 1F. (F-H) SUMOylation does not regulate Sgo1 dissociation from pericentromeres in response to tension. (F) Sgol expression is unchanged in metaphase-arrested sizl $\Delta$ siz $2 \Delta$ cells. Protein extracts from Figure $4 \mathrm{~F}$ were analysed by anti-HA and anti-Pgk1 (loading control) western blotting. (G) Sgol expression is unchanged in metaphasearrested sgol-2R, 4R and $5 R$ mutants. Protein extracts from Figure 4G were analysed by anti-Flag and anti-Kar2 (loading control) western blotting. The non-specific bands were marked with an asterisk. (H) Sgol localization in metaphase was unchanged in

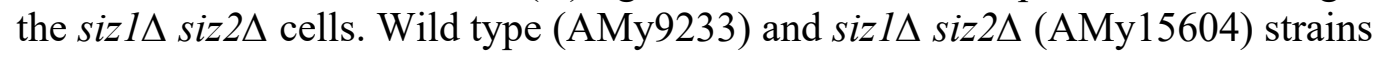
carry $p M E T-C D C 20, S G O 1-y e G F P$ and MTW1-tdTomato. Shown are representative pictures of cells. The point at which a bright Sgol focus was formed was assigned as time 0 . 

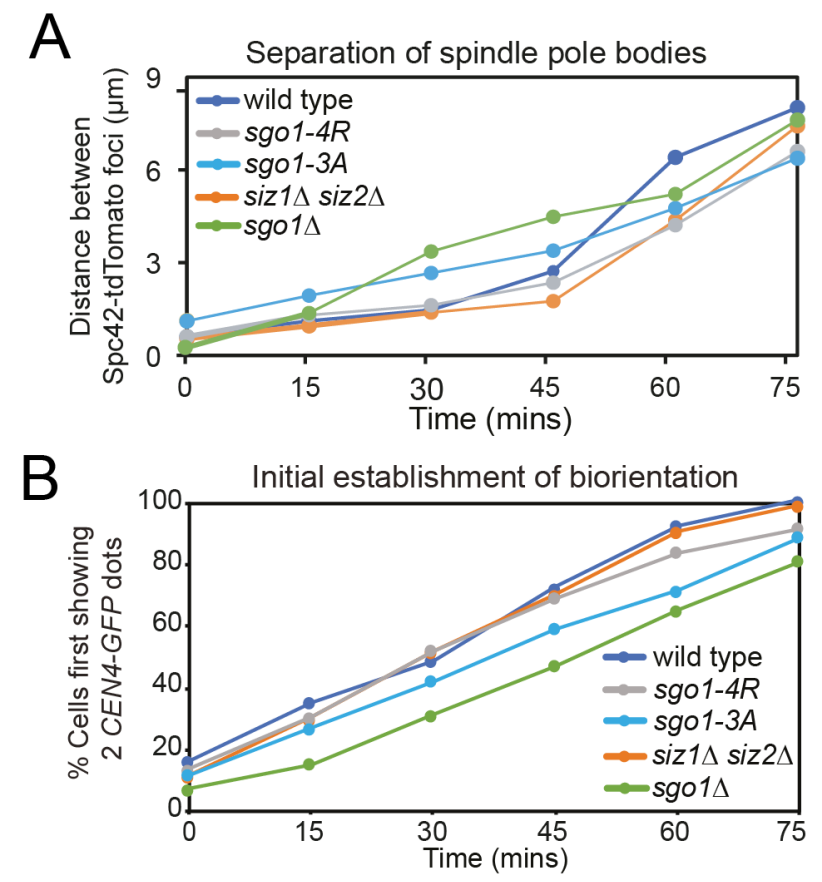

Figure S5, related to Figure 5. Sister kinetochore biorientation is proficient in SUMO mutants, but not in sgol or sgol-3A. Spindle pole body separation (A) and initial establishment of biorientation for the experiment shown in Figure 5C-D. (A) The distance between Spc42-tdTomato dots was measured by ImageJ and the average distance was calculated for each time point. (B) The initial establishment of biorientation is unaffected in siz $1 \Delta$ siz $2 \Delta$ and $\operatorname{sgol}-4 R$ cells. The time point at which a cell first displayed two CEN4-GFP dots was defined as the timing of the initial establishment of biorientation. 
A
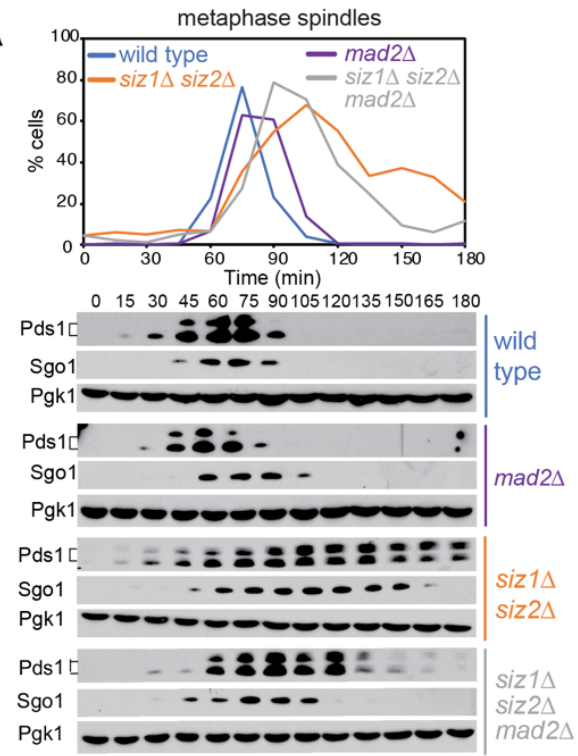

C
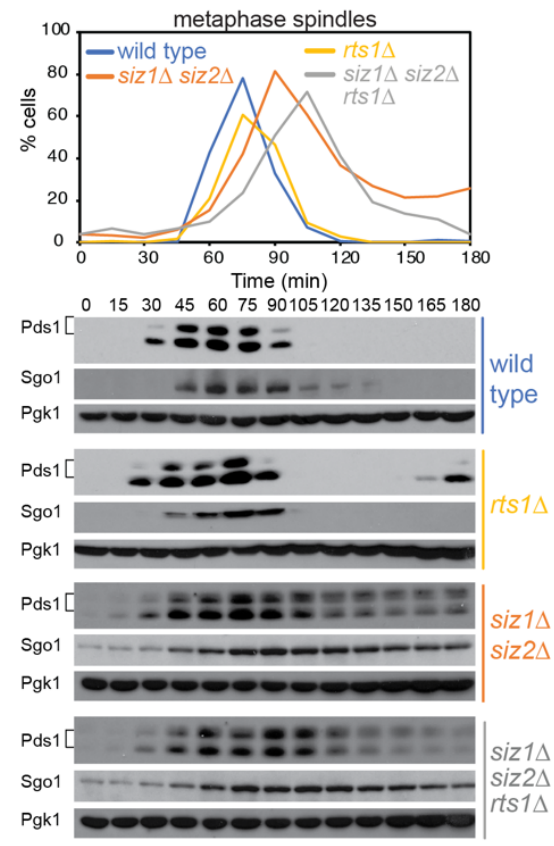

B
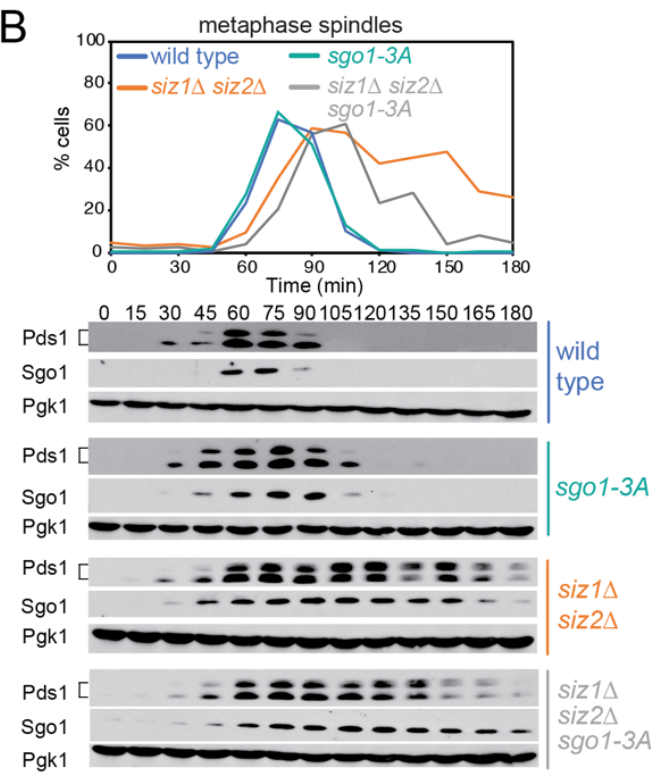

Figure S6, related to Figure 6. Inactivation of CPC and SAC, but not PP2A-Rts1, rescues the metaphase delay of $\operatorname{siz} 1 \Delta$ siz $2 \Delta$ cells. (A) Deletion of the SAC component, $M A D 2$, partially rescued the metaphase delay of siz1 $\Delta$ siz $2 \Delta$ mutant. Mitotic time course was performed as in (A) for the following strains: wild type (AMy8467),

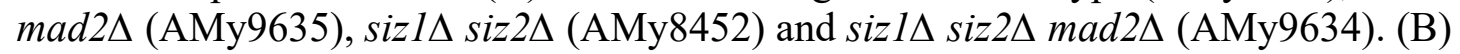
Partial rescue of the metaphase delay of $\operatorname{siz} 1 \Delta$ siz2 $\Delta$ cells by the sgol-3A mutation.

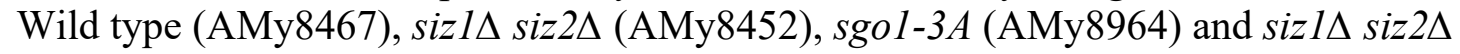
sgo1-3A (AMy8755) strains carrying PDS1-HA and SGO1-9MYC were analysed as described in Figure 1F. (C) Deletion of RTS1 did not rescue the metaphase delay of the siz1 1 siz2 $\Delta$ mutant. Mitotic time course was performed as in (A) for the following







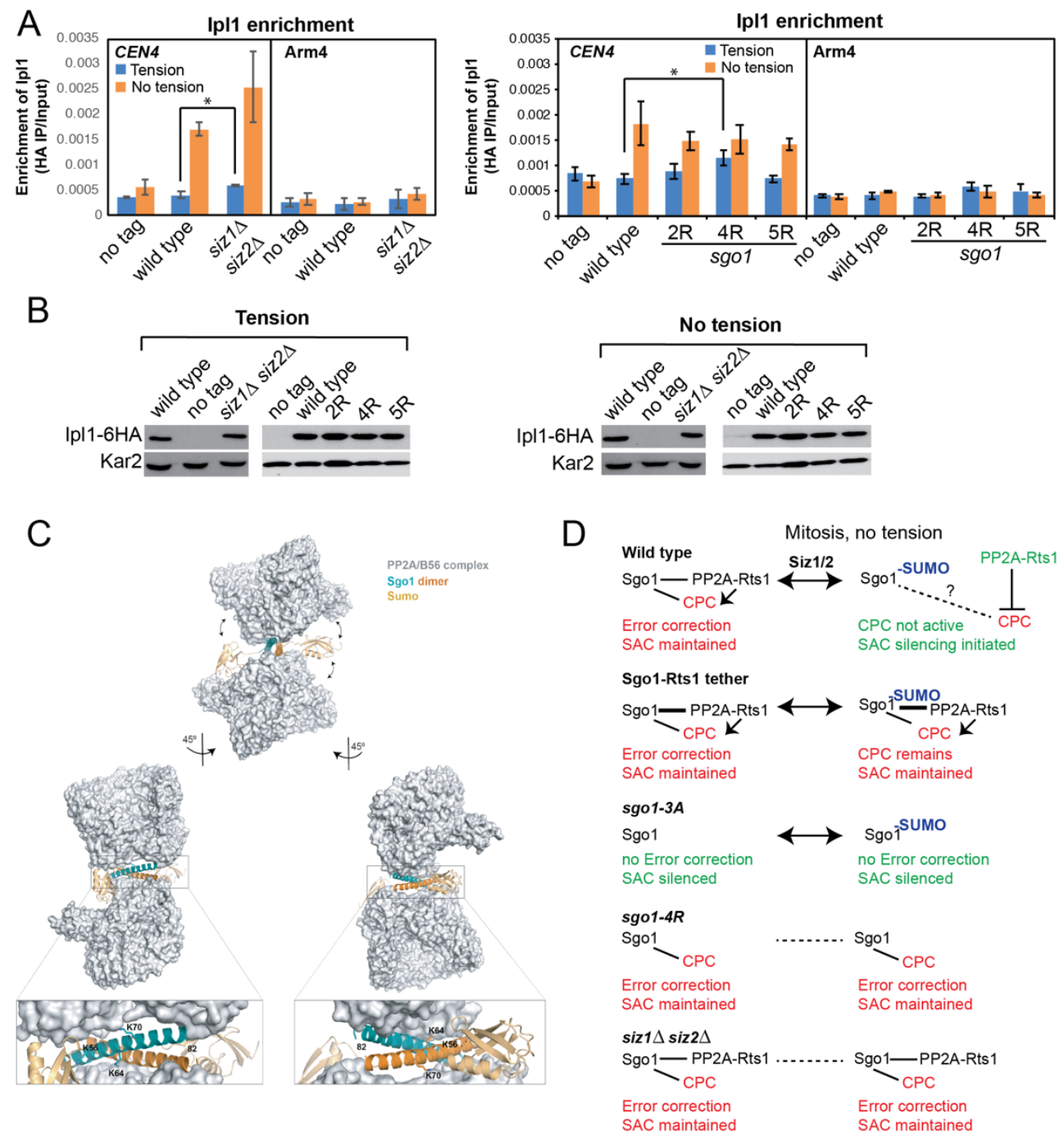

Figure S7, related to Figure 7. In Sgo1 SUMO-deficient cells, Ipl1 is not completely removed when the cells are under tension. (A) Ipl1 association with CEN4 and ARM4

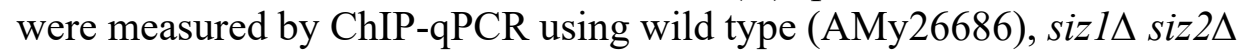
(AMy23194), sgol-2R (AMy26684), sgol-4R (AMy26692) and sgol-5R (AMy26691) carrying IPL1-6HA, together with a no tag control (AMy2508). Cells were arrested in metaphase by depletion of Cdc20 in the presence or absence of spindle tension. Error bars represent standard errors calculated from 5 biological repeats. ${ }^{*}=P<0.05$. (B) Ipl1 protein levels are unchanged in Sgo1 SUMO-deficient mutants. Protein extracts from (A) were analyzed by anti-HA and anti-Kar2 (loading control) western blotting. (C) Structural modelling predicts that SUMOylation on the coiled-coil domain of Sgo1 is incompatible with Sgo1-PP2A interaction. S.c. Sgo1PP2A interaction was modelled based on structural information obtained from cocrystallized human Sgo1(51-96) and PP2A using Phyre2 web portal (www.sbg.bio.ic.ac.uk/phyre2) [54]. Potential consequence of symoylation was modelled using the molecular graphic program PyMOL (The PyMOL Molecular Graphics System, Version 2.0 Schrödinger, LLC). According to this model, Lys64 and Lys70 are critically positioned at the binding surface with no room to accommodate a bulkier modification such as sumoylation. Lys56 is exposed to the solvent, but the attachment of SUMO (highlighted in gold) is expected to result in 
steric clashes with PP2A and weaken Sgo1-PP2A binding. Structural information is unavailable beyond Leu82 and so Lys 85 could not be included in this model. (D) Model for role of Sgo1 SUMOylation in stabilizing the bioriented state. In wild type cells, Sgo1 brings both PP2A-Rts1 and CPC to centromeres and PP2A-Rts1 enhances CPC localization. A minor pool of Sgo1 is dynamically SUMOylated and this both prevents PP2A-Rts1 binding and directly or indirectly promotes CPC removal, dampening its activity at kinetochores. Upon tethering of PP2A-Rts1 to Sgo1, release of PP2A-Rts 1 cannot occur and CPC activity persists. In sgol-3A cells, the interaction with both PP2A-Rts1 and CPC is absent and error correction is defective due to a failure to maintain CPC. In sgo1-4R cells, PP2A-Rts1, but not CPC binding is lost. The failure to SUMOylate, along with the absence of PP2A-Rts 1, means that the phosphatase cannot undergo its capture and release by Sgo1. Either as a consequence of this and/or other effects of Sgo1 SUMOylation, CPC is maintained on kinetochores resulting in ectopic error correction and destabilization of kinetochoremicrotubule attachments. siz1 $\mathbf{\Delta}$ siz $\mathbf{2} \Delta$ mutants show similar behaviour to sgo 1-4R except that Sgo1 is expected to retain the ability to bind PP2A-Rts1, so that absence of SUMOylation both prevents CPC removal and PP2A-Rts1 release. 